\title{
Single shot, nondestructive monitor for longitudinal subpicosecond bunch profile measurements with femtosecond resolution
}

\author{
I. V. Konoplev®, ${ }^{*}$ G. Doucas®, H. Harrison ${ }^{\dagger}{ }^{\dagger}$ A. J. Lancaster, ${ }^{\star}$ and H. Zhang \\ JAI, Department of Physics, DWB, University of Oxford, OX1 3RH, United Kingdom
}

(Received 30 September 2020; accepted 14 January 2021; published 2 February 2021)

\begin{abstract}
Accurate knowledge of the charged particle bunch longitudinal (time) profile is important in the context of wakefield accelerators, Compton Light sources, $\mathrm{x}$-ray SASE FELs and THz radiation sources. However, it is still a challenge to obtain this information for subpicosecond long bunches and microbunched beams with the required femtosecond (fs) resolution and nondestructively. Apart from determining the profile in a nondestructive manner, the ideal bunch diagnostic would enable extraction of all required information in a single shot, have a sufficiently high repetition rate to monitor each bunch, small footprint, good cost efficiency and reliability. In this paper we present the design of a longitudinal bunch profile monitor that can determine the charged particle bunch profile with femtosecond resolution, nondestructively and in a single shot via the spectral analysis of coherent Smith-Purcell radiation (cSPr). It is based on the simultaneous deployment of three gratings with different periodicities, each with its own set of detectors. The number of the frequency sampling points is equal to the number of the optical channels and the discrimination against background radiation is based on the different polarization properties of cSPr and background radiation. The rationales for the choices made to optimize the monitor operation will be presented and the criteria for determining the number of frequency sampling points will be discussed. The possible future developments of the monitor are also presented and discussed.
\end{abstract}

DOI: 10.1103/PhysRevAccelBeams.24.022801

\section{INTRODUCTION}

In the past few years there has been an exponential growth in the development of the next generation of particle accelerators that can be used as sources of coherent radiation, particle colliders and other research, industrial, and medical applications [1-6]. Most of these systems and applications generate charged particle bunches of femtosecond duration or require such bunches for successful operation [1-6]. An accurate (fs-resolution) knowledge of the longitudinal structure of the bunch is necessary in order to improve the understanding of the operation of the accelerator and improve its performance (e.g., FELs) or to optimize the system design and enable its operation within a predefined range of operating parameters. One typical example is that of the electron bunches generated in plasma based accelerators: these are short (a few fs long)

\footnotetext{
*Corresponding author.

ivan.konoplev@physics.ox.ac.uk

Tresent address: University of Cambridge.

Present address: National Physical Laboratory, Hampton Road, Teddington, Middlesex TW11 0LW, United Kingdom.

Published by the American Physical Society under the terms of the Creative Commons Attribution 4.0 International license. Further distribution of this work must maintain attribution to the author(s) and the published article's title, journal citation, and DOI.
}

microbunches, but their shot-to-shot stability is relatively low. Therefore, measurements carried out using either an average longitudinal bunch profile monitor [7-9] or a destructive method are neither accurate nor desirable and meaningful. In order to have a good understanding of the bunch formation and acceleration, a new generation of noninvasive, single shot beam profile monitors capable of providing femtosecond resolution for each bunch is required. There are several techniques that can potentially be used for longitudinal bunch profile measurements: (1) streak cameras [10] with resolution down to $200 \mathrm{fs}$; (2) electro-optical sampling [11] with resolution down to $60 \mathrm{fs}$; (3) coherent radiation spectral analysis [7-9,12-16], and (4) the gas-jet technique (based on the ionization of a neutral gas) [17]. All of them have their limitations and not all of them are nondestructive. Monitors based on coherent radiation spectral analysis have been shown to be reliable and in this paper the technique based on the spectral analysis of coherent Smith-Purcell will be considered.

Coherent Smith Purcell radiation (cSPr) is generated when a charged particle beam propagates in the vicinity (just above the surface) of a conductive periodic structure, such as a metallic grating $[7-9,18,19]$. The frequency range of the coherent radiation generated by subpicosecond and femtosecond beams (assuming that the beam does not intercept the target i.e., noninvasive regime) is in the low $\mathrm{THz}$ and FIR region. There are a number of other 


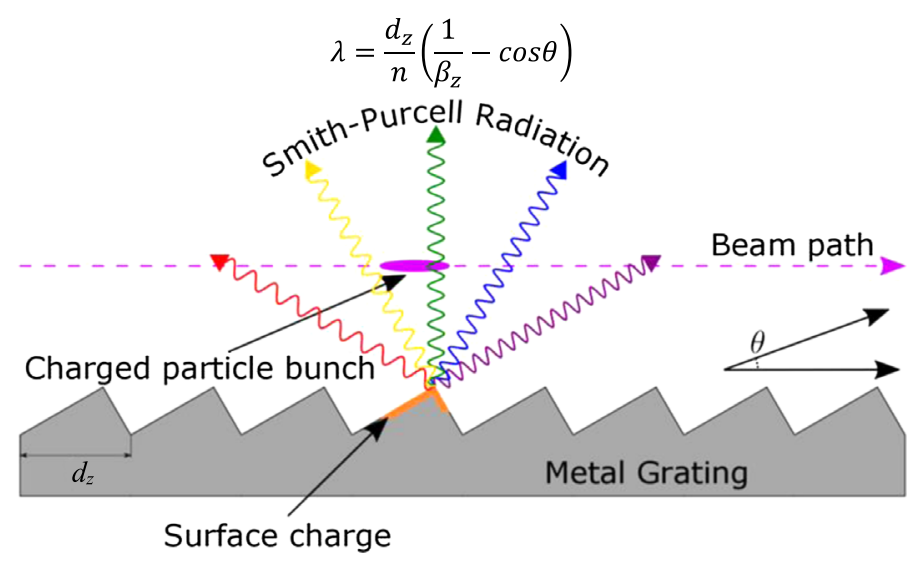

(a)

(b)

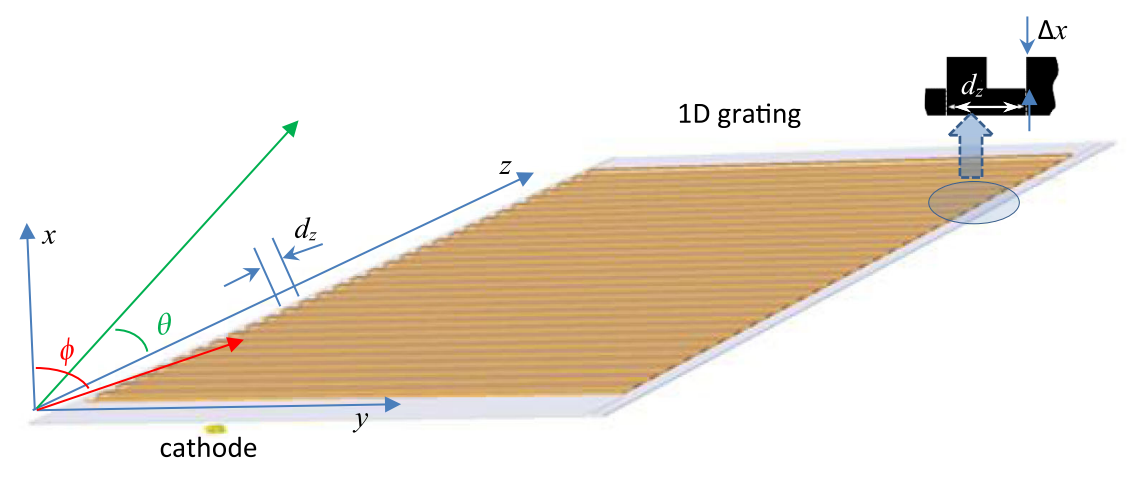

FIG. 1. (a) Schematic of a one-dimensional (1D) metal grating and coherent Smith-Purcell radiation generation via scattering of the surface charges and dispersion. (b) 3D numerical drawing of the 1D grating by MAGIC 3D software package used for the studies; the inset shows the grating profile.

mechanisms apart from cSPr that can be used to generate the coherent radiation: diffraction, transition, synchrotron, Cherenkov and FEL radiation. One of the disadvantages of these processes compared with Smith-Purcell is the need for additional vacuum lines to transfer the $\mathrm{THz}$ and FIR radiations to a set of spectrometers [12-16]. Although the radiation propagates in vacuum, there is inevitably signal loss and also several bunches are usually required to measure the full spectrum for the bunch profile reconstruction; i.e., these are not a single shot techniques. In the case of monitors based on Cherenkov radiation that use a dielectric slab for the generation of the radiation, there are additional drawbacks: the complex refractive index of dielectric materials in this frequency range (THz and FIR) is not well understood [20]; dielectric materials can degrade with time due to the harsh environment (x rays, high energy debris, etc.) prevailing inside accelerators; such materials may be a source of secondary electron emission affecting the vacuum and the system operation. The undulator radiation (FEL radiation) has advantages similar to that of the SP radiation: no target degradation, resolved issue with signal-noise separation and a well understood process; however, it requires external spectrometers and an expensive and comparatively long undulator (assuming a period of $1.5 \mathrm{~cm}$, one may require an undulator with at least 20-30 periods). On the other hand, the length of a grating needed to observe a measurable cSPr signal does not exceed a few centimeters. The grating itself operates as radiator and as an effective dispersive medium (Fig. 1) making unnecessary the use of external spectrometers. The use of a metal target makes it reliable and predictable, removing the disadvantages of Cherenkov based monitors. A cSPr based monitor can also be compact, with a footprint on the beam line of around 60 centimeters, depending on the monitor optics [7-9], reliable, vacuum safe for an accelerator facility and relatively economical.

In all schemes that rely on spectral analysis of the emitted radiation for the bunch profile reconstruction, the separation of the useful signal from the background noise is essential and one of the major challenges. The background signal is not only associated with the radiation generated up- or downstream from the monitor, it is also radiation generated from the apertures (diffraction radiation), target holders and other parts of the monitor itself that are exposed to the relativistic beam. This is a difficult problem and for a single shot monitor it can only be resolved, at the current 
stage, for monitors based on Smith-Purcell (SP) or undulator radiations.

In this paper we discuss the design of a single shot, noninvasive and nondestructive bunch profile cSPr monitor and its operational principles. We show the details of the technical design and discuss the rationale behind some of the design solutions. The paper is structured as follows: Sec. II is dedicated to an overview of the fundamental theory behind the cSPr monitor, developed and validated during a set of recent experiments [7,9,21]. In the same section we discuss briefly an experimental setup used to determine the average longitudinal bunch profile and show why it could not be transformed into a single shot monitor. Section III discusses the design of the single shot monitor and the justifications for some of the proposed solutions. In Sec. IV we discuss future steps of the monitor development and the potential for the design of a monitor capable of a full $3 \mathrm{D}$ bunch reconstruction and summarize the results.

\section{FUNDAMENTALS OF CSPr MONITOR FOR BUNCH LONGITUDINAL PROFILE RECONSTRUCTION}

Coherent Smith Purcell radiation (cSPr) is generated if an electron bunch propagates above a periodically patterned surface, for example a metal grating (Fig. 1). The gratings discussed in this paper are shallow, meaning that the amplitude of the corrugation $\Delta x$ [coordinates are defined in Fig. 1(b)] is small, less than a quarter of the generated wavelength $\lambda$, and the periodic patterns are machined on a metal surface that is assumed to be perfectly conducting. The grating is also assumed to be wide enough (over $1 \mathrm{~cm}$ ) compared with the beam transverse dimensions $\delta x, \delta y$ (around $100 \mu \mathrm{m}$ ) so that the grating boundary effects can be ignored in the first instance, allowing the "infinitely wide grating" approximation to be used in order to derive analytical solutions for the initial estimates of the yield.

In general, the periodic perturbations on the grating surface can be described as

$$
\begin{aligned}
x(z, y) & =x_{0}+\sum_{n, m} \Delta x_{n m} \cos \left(n \bar{k}_{z} z+m \bar{k}_{y} y\right), \\
\overrightarrow{\vec{k}} & =\overrightarrow{\vec{k}}_{z}+\overrightarrow{\bar{k}}_{y},
\end{aligned}
$$

where $\Delta x_{n m}$ is the amplitude of the $n, m$ harmonics and $\bar{k}_{z, y}=2 \pi / d_{z, y}, d_{z, y}$ are the period of the perturbations along the $y$ and $z$ coordinates. In Fig. 1(b) the 3D figure of the numerical model of the grating generated by 3D code MAGIC is shown. Here we consider that the grating is machined only along the beam propagation axis $z, n=1$ and $\bar{k}=\bar{k}_{z}$; i.e., it is parallel to the beam initial velocity. The use of the 1D periodic structures $\left(\bar{k}_{y}=0\right)$ is for the illustration of the model only; it is possible to consider the use of more complex periodic structures [18,19,22,23] which might reduce further the size and the mechanical complexity of the monitor [16] (see the Appendix). Figure 1(a) illustrates the dispersion of the cSPr; i.e., the dependence in the far field zone of the wavelength $\lambda(\theta)$ observed at an observation angle $\theta$. This is defined by relation (2):

$$
\lambda(\theta)=\frac{d_{z}}{n}\left(\frac{1}{\beta_{z}}-\cos \theta\right),
$$

where $\beta_{z} \cong \sqrt{1-\gamma^{-2}}$ is the longitudinal velocity of the charged particle normalized to the speed of the light $c$ (assuming small transverse velocities) and $\gamma$ is the Lorentz relativistic factor. The beam propagates in the vicinity of the grating and induces surface currents [Fig. 1(a)] on the facets. If the facet surface has no discontinuities the currents induced by the particle beam move with constant velocity and no propagating radiation is excited. On a continuous surface only evanescent fields, exponentially decaying from the conductor surface, defined by the "coupling parameter" or evanescent wavelength,

$$
\lambda_{e}=\frac{\beta \gamma \lambda}{2 \pi \sqrt{1+\beta^{2} \gamma^{2} \sin ^{2} \theta \sin ^{2} \phi}},
$$

are excited, where $\theta$ is the polar angle, i.e., in the plane defined by the beam trajectory and the normal to the trajectory crossing the grating surface, and $\phi$ is the azimuthal angle, i.e., in the plane perpendicular to the bean trajectory [Fig. 1(b)]. This field couples the charged particle beam and the surface currents, allowing energy transfer. The quasiparticle systems consisting of the surface currents and the evanescent waves are sometimes also known as plasmons. The surface perturbations/discontinuities, as defined by (1) and shown in Fig. 1, result in deceleration/scattering of the surface charges, leading to the generation of the propagating electromagnetic (EM) waves which radiate away from the grating. As a result, each perturbation on the surface is a radiator and taking into account the periodicity of the perturbations, and using Huygens construction, the fundamental cSPr dispersion relation (2) can be obtained. The first term in the bracket $\left(\frac{1}{\beta_{z}}\right)$ indicates only a time delay (in comparison with the speed of light) of each radiator excitation. There are two interesting points to be noted: (a) the radiating surface charges (plasmons), i.e., the free electrons in the induced surface charge "footprint" are not relativistic, although the footprint can "move" with superluminal velocity; (b) the dispersion relation (2) is defined for the far field zone, i.e., for observation distances $r>2 D^{2} / \lambda$ where $D$ is the largest dimension of the radiator. If this condition is not satisfied, then the radiation arriving at the detector will not be strictly monochromatic and this would have to be taken into account in the subsequent signal analysis [9]. It is desirable that the above inequality should define the minimal distance $r$ between the grating and the first optical element of the monitor. There is another useful property of coherent Smith-Purcell radiation that follows from the wavelength dependence on the longitudinal velocity $\beta_{z}$ in expression 

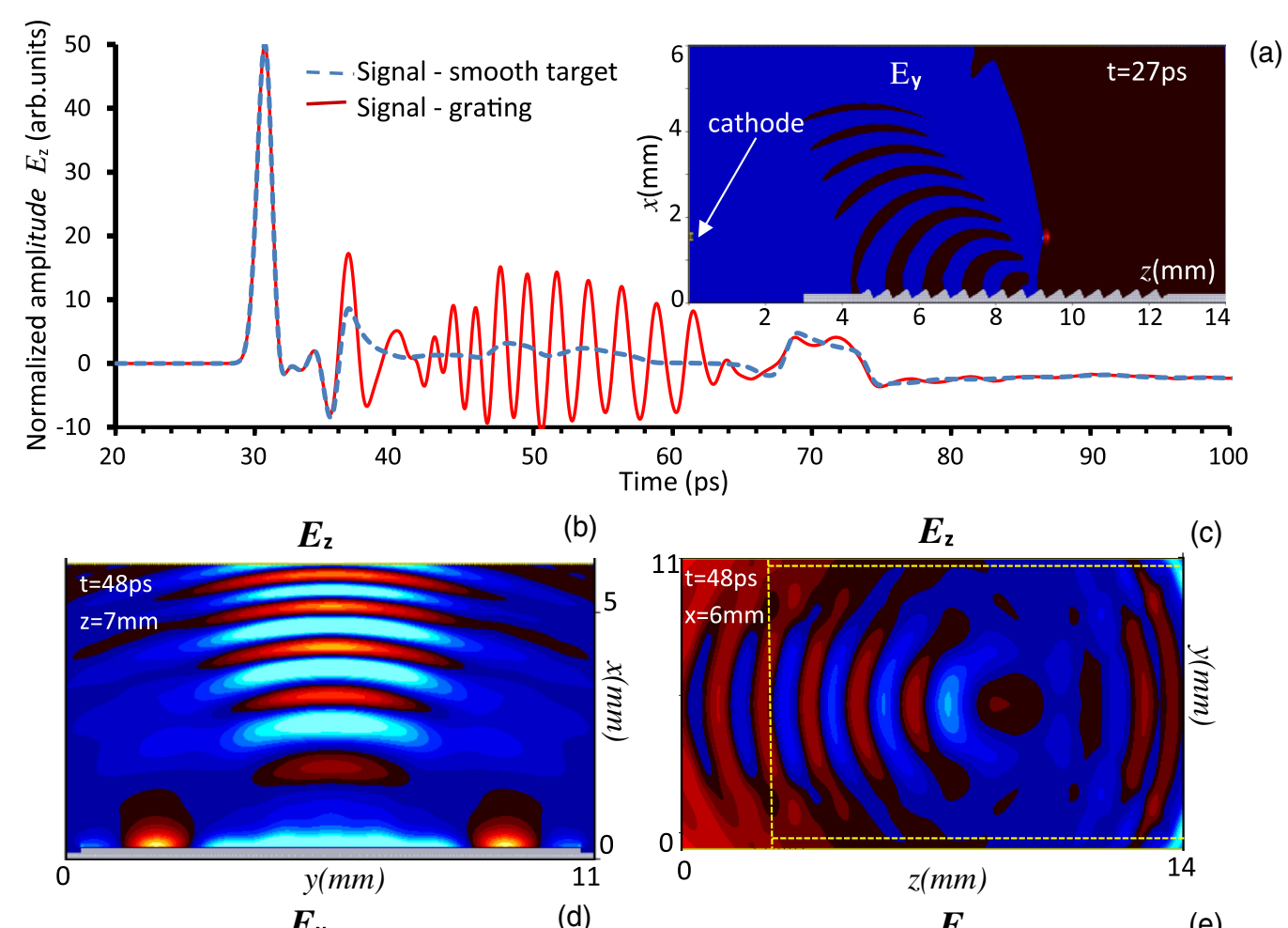

$\boldsymbol{E}_{\mathrm{z}}$

(c)
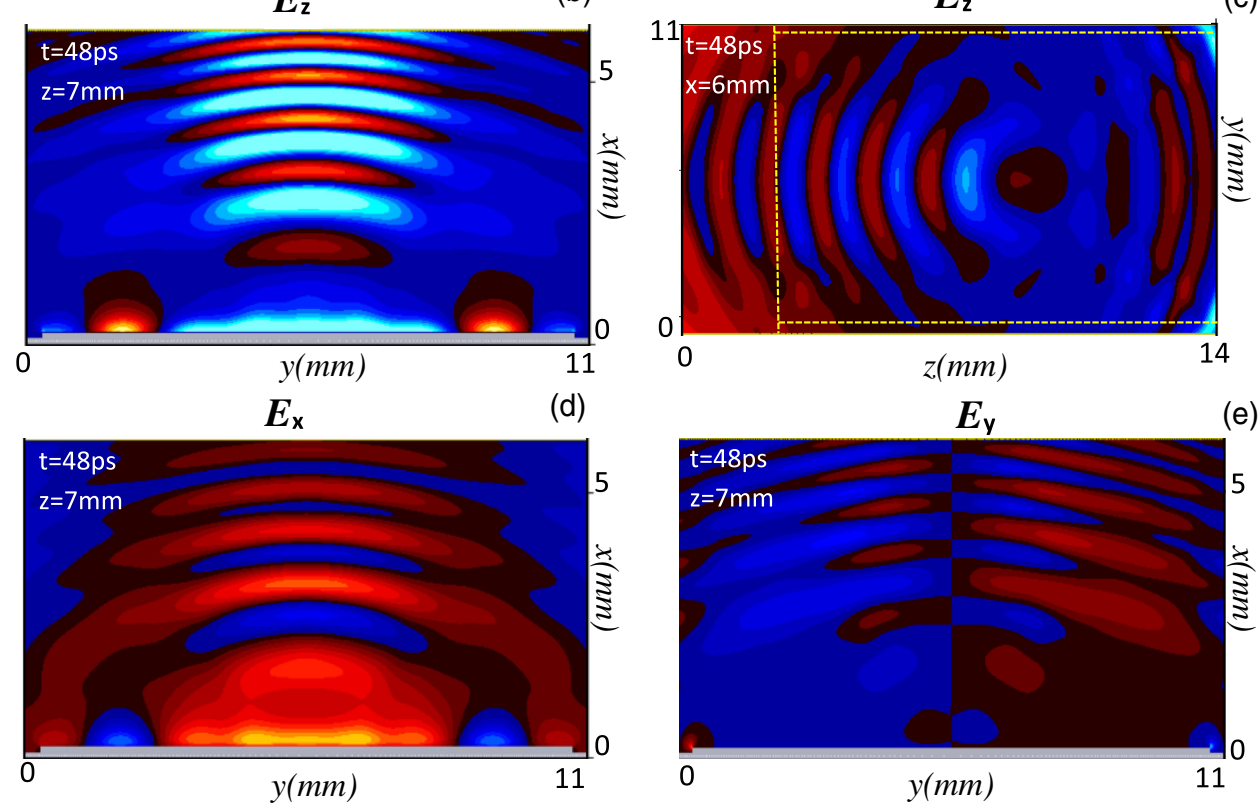

FIG. 2. The results of the numerical studies of the cSPr using 3D PIC code MAGIC showing: (a) the amplitude of the waveforms of the signals ( $E_{z}$ field component) generated by one-dimensional grating of $0.6 \mathrm{~mm}$ period (solid line) and smooth metal plate (dashed line) of the same geometry (blank target). The inset shows the grating and the contour plot of $E_{y}$ field component at $t=27 \mathrm{ps}$; (b)-(d) the contour plots of the electric field components $\left(E_{z, x, y}\right)$ showing the radiating field of cSPr generated by the $0.6 \mathrm{~mm}$ grating at $t=50 \mathrm{ps}$. The contour plots (b), (d) and (e) are observed for the $(x, y)$ cross section at $z=7 \mathrm{~mm}$ (middle of the grating). The formation of the side lobes (field variation along $y$ coordinate) is shown in parts (b) and (d). Part (c) shows the contour plot in the (y, z) plane, i.e., view from the top. The dashed line on (c) shows the borders of the target.

(2) namely the tolerance of the monitor to the spread of the energies inside the bunch:

$$
\delta \lambda=\frac{d_{z}}{n} \delta\left(\frac{1}{\beta_{z}}\right) \sim \delta \gamma / \gamma^{3}
$$

meaning that with an increase in the beam median energy the tolerance will also increase.

Figure 2(a) shows the waveforms generated by the $1 \mathrm{~mm}$ period grating (solid line) and a blank target (dashed line) if a $100 \mathrm{fs}$ electron bunch propagates $0.5 \mathrm{~mm}$ above them. The results shown were obtained using the 3D PIC code MAGIC $[19,24,25]$. The blank target, a smooth $\left(\Delta x_{\mathrm{nm}}=0\right)$ metal plate of the same geometry, has been suggested and used [7-9] in order to account for and subtract the background signals which are usually observed in any accelerator. The numerical data show the waveforms of the signals generated by the grating and the blank target. The EM radiation generated by the grating consists of the cSPr and the background signals while the blank target does not produce the cSPr signal. The geometrical parameters of the numerical box used for these studies were: width $11 \mathrm{~mm}$ (the grating width was $10 \mathrm{~mm}$ ); height $6 \mathrm{~mm}$ and total length $14 \mathrm{~mm}$. The electron beam transit time through the numerical box is around $50 \mathrm{ps}$. The contour plot shown in the inset of Fig. 2(a) is taken at $t=27$ ps i.e., when the bunch position was at around the middle of the grating. The contour plots of the radiated electric field components 
shown in Figs. 2(b)-2(e) were taken at 48 ps at different cross sections, i.e., with no electron bunch in the numerical box. In Figs. 2(b), 2(d), and 2(e), the contour plots of the electric field components are shown in the $(x, y)$ plane at the middle of the grating and perpendicular to the beam direction propagation, while Fig. 2(c) shows the $E_{z}$ field contour plot observed from the above of the target (the boundary is indicated by the dashed line). The wave forms show that the cSPr radiation will be radiated for approximately 25 ps, i.e., from 40 to 65 ps, Fig. 2(a), which is the transit time of the bunch above the grating. The wave form in Fig. 2(a) has also a frequency "chirp," illustrating indirectly the expression (2).

In the accelerator environment the background contribution could be coming from anywhere while in simulations this contribution is a rather well understood phenomenon and can be used to underline the challenges. The background radiation peaks [Fig. 2(a)] are associated with the diffraction radiation and transition radiation due to launch of the electron beam into the numerical box and the target's front and back ends; the peaks can be identified on both traces. It is important to note that the energy content and the amplitudes of the field due to background radiation are comparable with the cSPr generated by the grating. This underlines the fact that background noise has to be subtracted from the total signal in order to determine accurately the true SP signal and hence the bunch length. Figures 2(b)-2(e) also illustrate the directionality of the cSPr. This is an important feature for the design of the bunch profile monitor [7-9]. There are factors affecting the directionality (azimuthal dispersion) of the cSPr. Due to the finite width of the grating $L_{w}$, there is excitation of side lobes, as shown by the variations of the $E_{z, x}$ and the finite opening angle of the main lobe. The directionality of the main signal is within a small opening angle if $L_{w} \gg \lambda$ i.e. $\tan (\delta \hat{\varphi}) \sim \lambda / 2 L_{w}$, which in this case corresponds to an angle of less than 6 degrees. The directionality of cSPr is also defined by the dependence of the $\lambda_{e}$ on the relativistic factor $\gamma$ (3) as only when $\phi$ is small and finite i.e., $\phi<1 / \beta \gamma$ can strong coupling takes place:

$$
\lambda_{e} \cong \frac{\beta \gamma \lambda}{2 \pi} .
$$

These are two factors affecting the azimuthal dispersion of the cSPr and should be considered during the design. In cases where a highly relativistic beam $(\gamma \gg 1)$ is monitored the geometrical factor i.e., the finite width of the grating will be the definitive factor.

A multigrating average bunch profile monitor has been constructed and used [7-9]. The gratings were mounted on a carousel, as shown in the inset of Fig. 3(a); this allowed the sequential exposure of three gratings, of different periodicity, and a blank in order to measure the total and the background radiation spectra, respectively. The use of

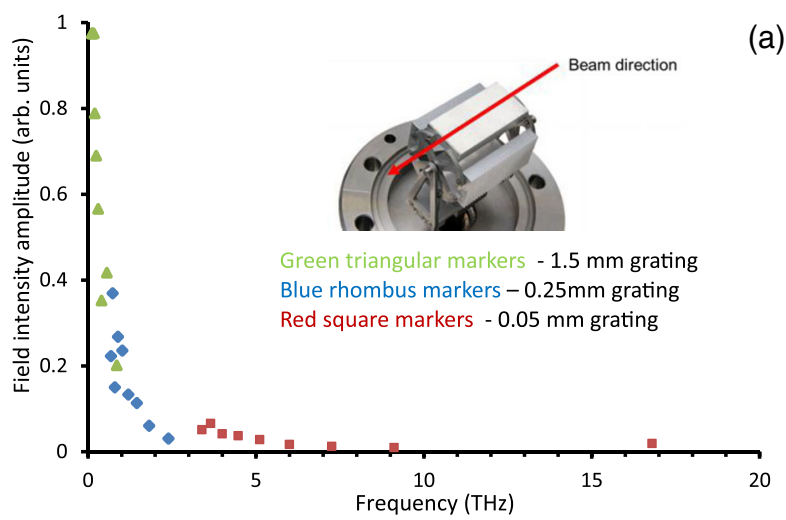

(a)

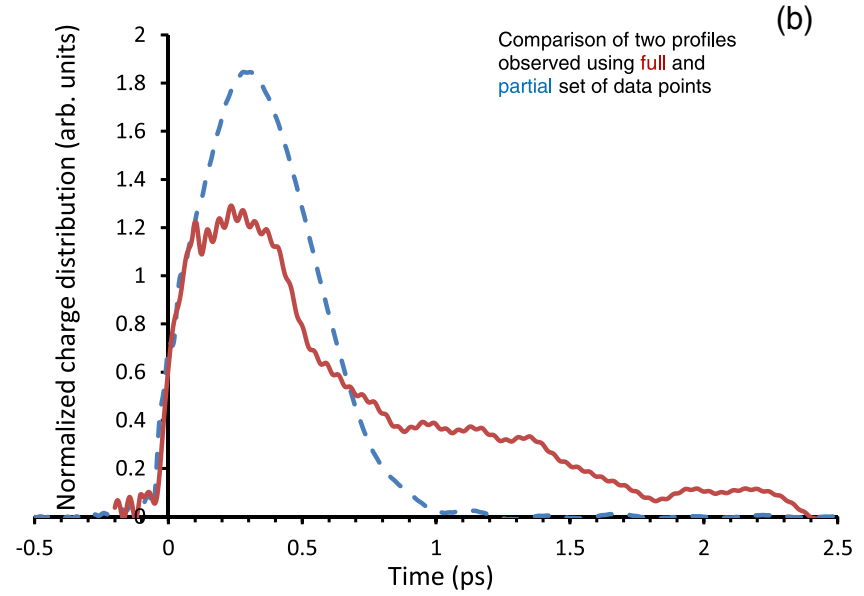

FIG. 3. The cSPr spectrum (a) and reconstructed bunch profile (b) observed using the average bunch profile cSPr monitor. The inset to (a) is the photograph of the four-target carousel used in the average bunch profile cSPr monitor with the red arrow showing the direction of electron beam propagation. In (a) the dots represent signal intensities at different sampling frequencies measured using the $1.5 \mathrm{~mm}$ grating (triangular marks), $0.25 \mathrm{~mm}$ grating (rhombus marks) and $0.05 \mathrm{~mm}$ grating (square marks). In (b) the profile shown by the dashed line was reconstructed by truncating all the data below $0.5 \mathrm{THz}$ and substituting them with a Gaussian extrapolation while the profile shown by the solid line was obtained by using the full set of data.

three gratings enabled the sampling of 33 frequencies over a very broad wavelength range [Fig. 3(a)] from $2649 \mu \mathrm{m}(0.11 \mathrm{THz})$ to $18 \mu \mathrm{m}(17 \mathrm{THz})$, while the total spectrum was completed via extrapolation and interpolation techniques [7-9]. In Fig. 3(b) the reconstructed bunch profile is shown by the bold line. These studies showed the possibility of the profile reconstruction with femtosecond resolution and the importance of measuring the spectrum as accurately as possible both at high frequencies, above $1 \mathrm{THz}$, and in the low frequency range i.e., below 0.5 THz. Figure 3(b) illustrates two profiles: the dashed line graph has been generated from a spectrum where all the experimental points below $0.5 \mathrm{THz}$ have been removed and replaced by extrapolation from $0.5 \mathrm{THz}$ to 0 by means of a Gaussian function; the solid line profile has 
been generated using all the available data points. This set of recent experiments [7-9] resulted in a better understanding of the fundamental properties of cSPr and stimulated the next step-the development of the single shot monitor.

\section{OPERATION AND THE DESIGN OF THE SINGLE SHOT CSPr BUNCH PROFILE MONITOR}

In this section we outline and discuss the conceptual design and operation of a cSPr monitor capable of measuring the time profile of subpicosecond (femtosecond
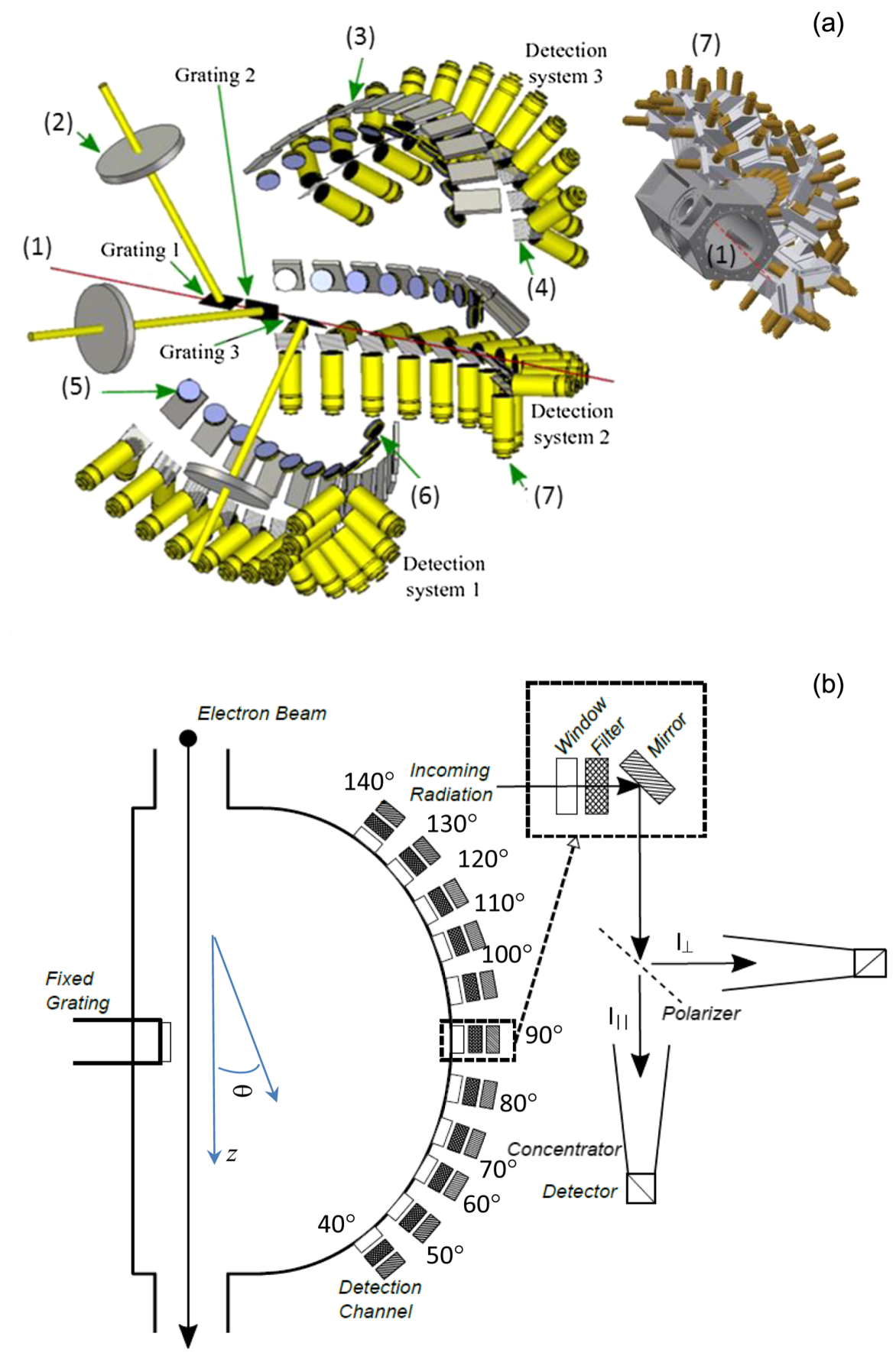

FIG. 4. (a) The 3D technical drawing of the 3 grating monitor (the vacuum chamber is transparent for clarity) with the inset showing the vacuum chamber. The electron beam trajectory is shown by line (1), vacuum flanges (2) support the movable rods which are used to adjust the gratings' positions, the mirrors (3) redirect the signal from the targets to the polarization splitters (4). The radiation emerges through the silicon windows (5) and narrow passband filters (6) can be used to filter unwanted frequencies. The signals are measured by a detection system consisting of detectors (7) and Winston cones (8). The inset illustrates the vacuum chamber with the vacuum ports, detectors (7) and beam trajectory (1); (b) the schematic of the cSPr monitor. The background and cSPr signals are separated via polarization measurements, as shown in the inset. 
resolution) charged particle bunches or trains of microbunches, nondestructively and in a single shot, almost in real time. The monitor is based on a stationary set of gratings and utilizes the polarization properties of cSPr to separate the signal from the background radiation. The results/principles presented can be used to construct a diagnostic device capable of monitoring either the basic longitudinal parameters of the bunch, such as Gaussian half-width half maximum, or the periodicity, modulation depth of a microbunch train or to obtain detailed information about the longitudinal profile of a single bunch with femtosecond resolution $[9,26]$. To cover as wide frequency range as required, it is proposed to deploy several gratings simultaneously. Figure 4(a) shows the 3D design of a system with three gratings, while Fig. 4(b) illustrates a schematic of the detector arrangement around one of the gratings. All the gratings are exposed to the same bunch and in order to avoid cross-talk between neighboring gratings, the gratings and their detector arcs are rotated sequentially by $30^{\circ}$ around the beam. The inset of Fig. 4(a) shows the design of the vacuum chamber, the position of the detectors and the technical ports. The design is based on three $40 \mathrm{~mm}$ long gratings, and for our studies gratings of periods $1.2,0.5$ and $0.1 \mathrm{~mm}$, covering the wavelength range from 2 to $0.02 \mathrm{~mm}$ with 33 sampling frequencies, were used. The output channels have a diameter of $20 \mathrm{~mm}$ and are spaced at $10^{\circ}$ angular intervals in the range $40^{\circ}$ to $140^{\circ}$ [Fig. 4(b)]. All detectors are located along arcs of radius around $400 \mathrm{~mm}$ from the gratings and the centers of the arc are linearly shifted from each other by the length of the grating $(40 \mathrm{~mm})$. The inset of Fig. 4(b) shows that each channel will have a beam splitter and two detectors in order to determine the degree of polarization (DoP) of the incoming radiation, thus increasing the complexity of the monitor system [Fig. 4(a)]. The monitor shown allows 33 frequency sampling points and to carry out the measurement the use of 66 detectors will be required. This number could be changed, as will be discussed below, and we note again that the new design has stationary (nonrotating) gratings.

One of the important requirements for the accurate determination of the longitudinal profile is the subtraction from the measured signal of any background contribution. Previously this was done by using a blank target. However, this prevented the transformation of the average bunch profile monitor into a truly single shot instrument. The results of the numerical studies, using 3D PIC MAGIC [24], which are shown in Figs. 5(a)-5(c), illustrate this. In Figs. 5(a) and 5(b) the frequency dependence of the amplitudes of the $E$-field components are shown before (cSPr signal with background radiation) and after (pure cSPr signal) the subtraction, respectively, of the background signal. The initial signal [Fig. 5(a)] has very different spectral structure as compared with cSPr signal [Fig. 5(b)] and using it would lead to erroneous pulse profile. The property which allows one to overcome this challenge and separate the cSPr from the background, while performing single shot measurements, is polarization. The results of the simulations shown in Fig. 5(c) illustrate that the degree of the polarization (DoP) is nearly $100 \%$ at small azimuthal angles $\phi<1 / \beta \gamma$. As a result, the well-defined cSPr polarization may allow differentiation of the background and cSPr signals; this also obviates the need for the use of a blank target. However, the blanks will still be required during the monitor installation and initial tests in order to determine the DoP of the background radiation.

\section{A. Coherent Smith-Purcell radiation}

In order to reconstruct as accurately as possible the temporal profile of the bunch, it is necessary to have measurements over a wide frequency range; this can be achieved by using several gratings as shown in Fig. 4. The gratings are located at the end of drive mechanisms which allow for adjustment of the beam-grating separation as well as providing a mechanism for retraction of the gratings. It is worth recalling that the EM energy radiated, as cSPr, in the solid angle $d \Omega$ and the frequency interval $d \omega$ by the interaction of a single electron with a grating depends on the electron- grating separation $h_{0}$ :

$\left(\frac{d^{2} I}{d \omega d \Omega}\right)_{1}=e^{2} \frac{L_{g r}^{2}}{c d_{z}^{3}} \frac{\lambda n^{3} \beta^{3}}{(1-\beta \cos \theta)^{3}} R^{2} \exp \left[-\frac{2 h_{0}}{\lambda_{e}}\right]$,

where $n$ is the harmonic number and $R^{2}$ is the dimensionless "efficiency" of the grating [7-9]. To observe efficient coupling to the grating, the distance $h_{0}$ should be less than the evanescent wavelength $\lambda_{e}$ (3). Expression (3), which defines $\lambda_{e}$, shows that the distance $h_{0}$ is scalable with the radiating wavelength and the bunch energy. This defines an optimal distance for each grating taking into account the grating period and the bunch energy. For example, for an effective excitation of radiation at $1 \mathrm{THz}$ by a $10 \mathrm{MeV}$ electron, the distance between particle and grating should be $1 \mathrm{~mm}$ or smaller.

The cSPr generated by an electron bunch consists of coherent $S_{\text {coh }}$ and incoherent $S_{\text {inc }}$ components which reflect the distribution of the electrons inside the bunch. The expression for the total energy generated by a bunch of $N$ electrons is

$\left(\frac{d^{2} I}{d \omega d \Omega}\right)_{\text {total }}=\left(\frac{d^{2} I}{d \omega d \Omega}\right)_{1}\left[N S_{\text {inc }}+N(N-1) S_{\mathrm{coh}}\right]$,

where $N$ is the total number of the electrons in the bunch. The incoherent component is proportional to $N$ and dominates at wavelengths that are significantly shorter than the bunch length or shorter than any internal features of the bunch. The coherent component is proportional to $N^{2}$ and it encodes the temporal profile of the bunch. It is 

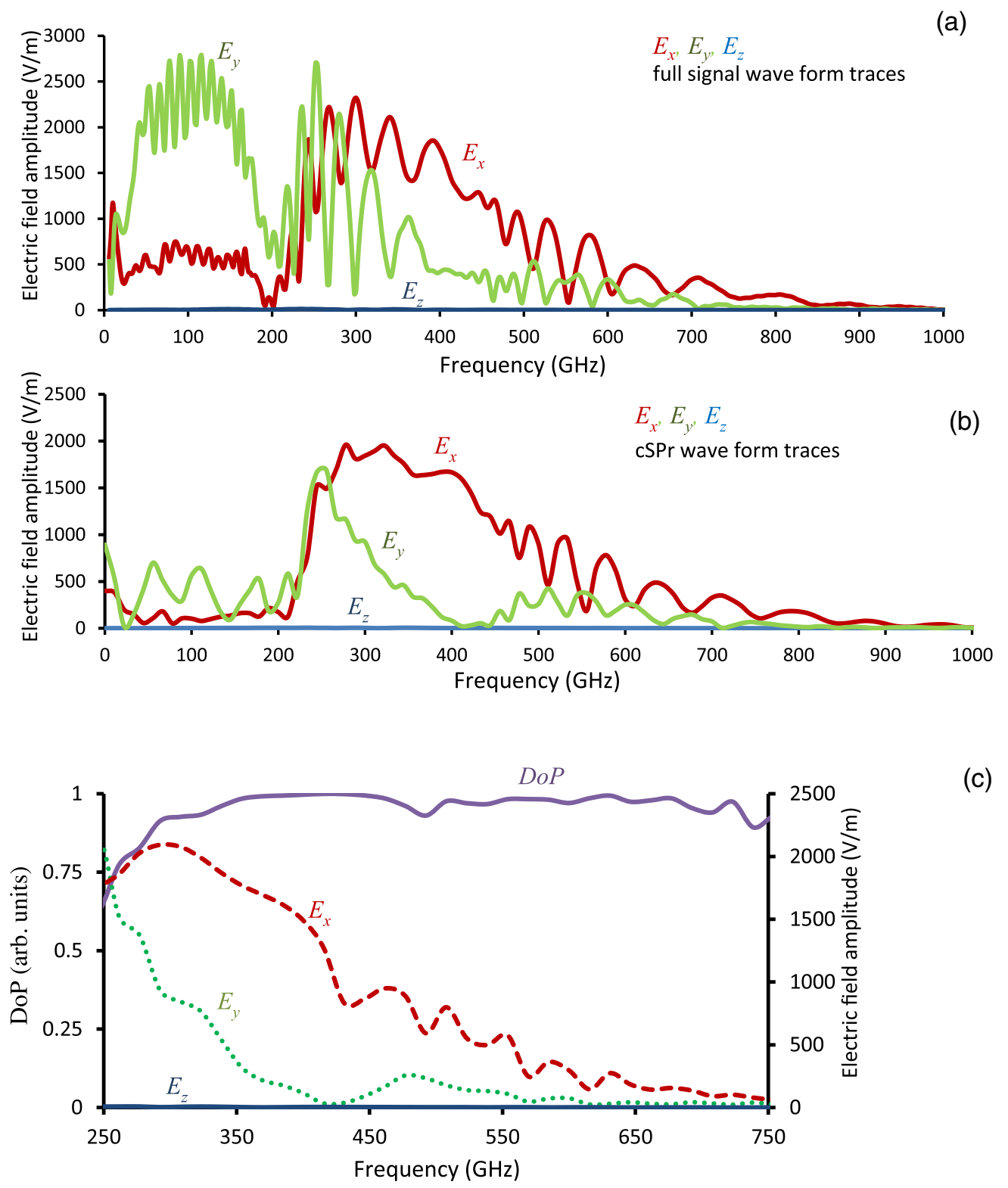

(c)

FIG. 5. The results of cSPr DoP studies using 3D PIC MAGIC: (a) the spectra of the field components $\left(E_{z, x, y}\right)$ of the full signal and (b) the spectra of the field components $\left(E_{z, x, y}\right)$ of the cSPr signal. The comparison of the spectra of the degree of polarization and the cSPr field components is shown in (c).

important to have accurate measurements both at high frequencies, through the use of small period gratings, in order to recover the fine features of the bunch and at low frequencies (generated by long period gratings) in order to determine the overall length of the bunch. Hence, in order to cover the broad frequency range needed it is advisable to use at least three gratings of different periods.

The expression (7) is valid for a grating of infinite width and understanding the effect of finite width on the energy generation is important. Figure 6 illustrates this dependence by comparing the radiated energy from an infinite width grating and a grating of finite width $L_{w}$. A grating made of ideal conductor of length $60 \mathrm{~mm}$ and periodicity of $1 \mathrm{~mm}$ was considered in this example. A Gaussian bunch of $\sigma_{x, y, z}=100 \mu \mathrm{m}, 1.2 \mathrm{nC}$ charge, $20.35 \mathrm{GeV}$ energy and propagating at around $1 \mathrm{~mm}$ above the grating was used to stimulate cSPr. For the given bunch transverse parameters, the $20 \mathrm{~mm}$ wide grating, as used in the average bunch profile monitor, would generate around $60 \%$ of energy expected from an infinite grating while a $50 \mathrm{~mm}$ wide grating will produce $90 \%$ of the expected signal at this energy. Therefore, it is advisable to use gratings that are as wide as possible as compared with the beam's $\sigma_{x, y}$ and to bring the beam as close to the grating as possible in order to minimize the interference from the grating boundaries [Fig. 2(b)]. The design of the 


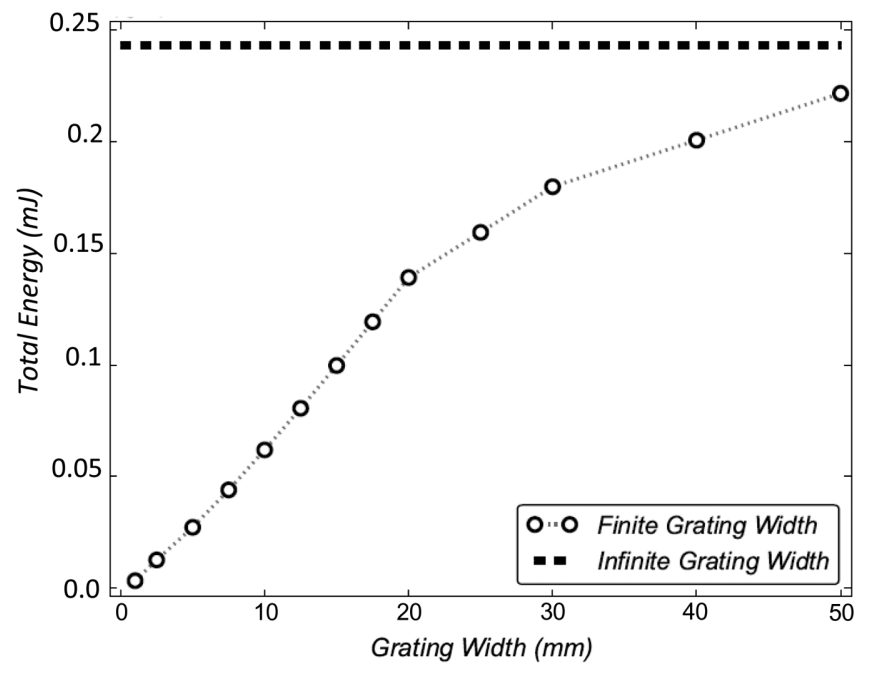

FIG. 6. Comparison of the signal intensity from the gratings of infinite (dashed line) and finite (dotted line) widths.

surface periodic patterns (grating) is also discussed in the Appendix.

\section{B. Vacuum chamber}

The vacuum chamber [see inset of Fig. 4(a)] with its technical and output optical ports is an essential component of the instrument [27] and its design mast take into consideration a number of challenges. All the ports of the monitor must be vacuum sealed, while the input and output beam ports are directly connected to the beam channel. To minimize the number of ports the most obvious design of the vacuum chamber would have been a barreltype chamber [16] with a large flat output window. This design, however, leads to a number of challenges, which could result in erroneous measurements. In such a design the cSPr would not impinge at $90^{\circ}$ to the surface of the finite width window and unless the window width is much thinner than the observation wavelength a significant refraction could occur, resulting in part of the radiation missing the detectors. The second potential problem follows from the fact that the dispersion relation (2) holds true only for radiation propagating in vacuum and unless the vacuum chamber is large, the vacuum window will affect the dispersion relation (2) causing diffraction and resulting in more complex dispersion. Another problem is that the necessarily thick window will affect the degree of polarization of the non-normal incident radiation due to birefringence effects and distort the measurements. Consequently, we suggest that the chamber has collecting optics positioned along the arc as shown in Fig. 4. Coherent Smith-Purcell radiation propagates radially from the gratings. Positioning the optic channels along the arc, with the vertices at the center of the grating, leads to approximately normal incidence of the rays on the output windows for all frequencies, minimizing refraction and preventing the birefringence effects. This geometry adds the possibility of background radiation filtering, if narrow passband filters and small angle acceptance optics are used [28,29]. As discussed earlier, meeting the far field zone condition is important, and in the case of a monitor with several gratings, the radii of the arcs $r_{i}$ must be $r_{i} \geq \frac{2 L_{g r, i}^{2}}{\lambda_{i}}$ where $L_{g r, i}$ is the length of the grating with index $i$. This defines and limits two parameters: the minimal radii of the arc of the chamber which would allow the interference to take place in the free space prior to the signal collection and the dimensions of the gratings (normally this will be the length of the grating) in order to keep the arcs' radii small and the monitor compact.

Finally we note that the careful design of the vacuum chamber is essential to reduce the background radiation signal. There are several possible sources of the background radiation e.g., the wakefields generated by the beam at the input, output and inside of the chamber. In the case of a high level background radiation one may consider additional steps to reduce it, for example by installing a large window opposite the gratings and the output arc together with an absorbing material.

\section{Detectors, polarizers and signal collecting optics (concentrators)}

The monitor's EM radiation collection system can be separated into three subsystems: signal receiving or guiding, signal polarization separation and signal detection. The design of each subsystem is dependent upon the specific parameters of the particle accelerator and the intended use of the monitor. As noted in the previous sections, the average bunch profile monitor used eleven channels covering a broad frequency range by sampling the spectrum at 33 frequencies (11 from each grating) [7,9]. The relatively low sensitivity integrating pyroelectric detectors, which were used previously to measure the cSPr signal, required energies in the $\mu \mathrm{J}$ range before they could detect the signal. Narrow band waveguide array plate (WAP) filters were used in this system to filter spurious frequencies [28] with nonimaging light concentrators (Winston cones) [29] and with small solid angle acceptance. The disadvantages of this are clear and associated with the following challenges: the required detectors (and filters) must have consistent performance (response/sensitivity /transmission /acceptance) over the frequency range of interest $(0.1 \mathrm{THz}$ to $10 \mathrm{THz}$ ); calibration and signal loss as a result of using WAP filters; low sensitivity due to the use of broadband detectors.

\section{Detectors}

To overcome the challenges, the use of dedicated signal collecting arcs for each of the three gratings is suggested. Each arc, and its associated detection channels, are specially designed for the expected frequency range. The use 
of the WAP filters is avoided in favor of waveguide filters such as Bragg filters [22] with nearly $100 \%$ transmission of the selected frequency. The proposed system allows the selection of the best available detectors for each specific sampling frequency, without being necessarily limited to pyroelectric detectors, though these detectors cover a broad wavelength range, from around $2 \mathrm{~mm}$ to $10 \mu \mathrm{m}$, and are cheap and robust. Alternatively it is possible to use InGaAs Schottky diode based detectors [30], which have a much narrower wavelength range [0.1 to $3 \mathrm{~mm}$ ] but are much faster and more sensitive. The application of the Schottky diode based detectors opens up the possibility to use the monitor for low energy (below $100 \mathrm{MeV}$ ) electron accelerators, capable of generating low charge $(<50 \mathrm{pC})$, short ( $<50 \mathrm{fs}$ ) electron bunches. Such experiments with the low energy accelerators were carried out at KEK and Tsinghua University [21,26] and demonstrated the possibility of using such detectors to measure cSPr signals. The use of the waveguide mounted Schottky barrier diode (SBD) detectors [30] reduces the low frequency noise, hence making low frequency filtering redundant. However, these detectors have limitations at high frequencies, as their sensitivity decreases significantly at frequencies above $3 \mathrm{THz}$; only energy integrating detectors, such as pyroelectric detectors or bolometers, have an acceptably flat response and reasonable sensitivity. The low temperature detectors like bolometers are bulky and difficult to use in a closed, space-limited accelerator environment and as a result the pyroelectric detectors can be considered as a good option. Ultimately, the choice of the detector is dictated by the intended application of the monitor. This will also define the design of the signal receiving and guiding optics which deliver the radiation to the detectors. Whatever detector system is used, it will be necessary to determine the responsivity of each detector at the corresponding wavelength. This is a task which is essential for all beam profile monitors that are based on measurements in the frequency domain.

\section{Receiving and guiding system}

Due to the low intensity of the cSPr signal, each detector has to have a signal receiving/collecting and guiding system. In the case of the waveguide-mounted SB detectors it is expected that the waveguide optics will consist of horns, possibly a low loss spherical lens, narrow band filters and waveguides. In the case of pyroelectric detectors nonimaging optics, like a Winston cone, can be used to enhance the signal. If Winston cones are used it is important to locate the detector as close to the cone's vertex as possible in order to avoid losses. It is noted that the Winston cones shown in all the figures of this paper are for illustrative purposes only. As mentioned earlier, the use of the waveguide SBD detectors is advantageous as the waveguide ensures low frequency filtering without the need for additional filters, thus simplifying the detection system. If filtering is required it is possible to use other types of filters such as narrow band Bragg filters, which are polarization sensitive and have better transmission characteristics at the frequency of the interest.

\section{The cSPr signal and background separation system}

To separate background radiation from the cSPr signal using their relative polarization, a system capable of splitting the signal into components with different polarizations will be required. In Fig. 7, the technical drawing of the cSPr signal background radiation separation system is shown. This consists of an input window, a signal redirecting mirror, which is necessary in order to locate the detectors behind lead blocks and screen them from possible ambient $\mathrm{x}$-ray radiation, a polarizer which operates as a beam splitter and the detectors. The arrows indicate the path of the radiation signal. Freestanding metal wire grid polarizers (WGP) [31-33] are proposed for this system (Fig. 9) as the transmission of such polarizers is above $95 \%$ for all of the spectrum that is of interest. These polarizers have wires that are supported only by a frame, without a dielectric substrate, thus avoiding the dielectric-signal interference. They are commercially available and are neither prohibitively expensive, nor too fragile; their frequency response is largely governed by the interaction of the wire grid with the incident radiation [31-33]. In the low frequency range (up to $1 \mathrm{THz}$ ) we suggest the use of tungsten WGPs with $30 \mu \mathrm{m}$ period and $10 \mu \mathrm{m}$ diameter

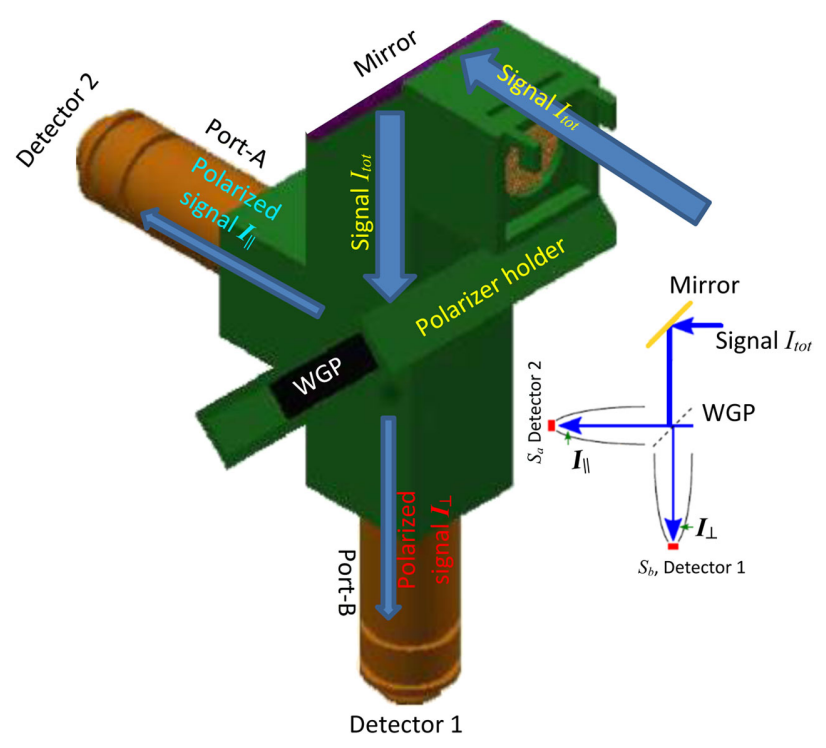

FIG. 7. Detailed technical drawing of an optical channel with all parts and the beam ray path shown. The inset is the schematic diagram of the channel in order to clarify some of the details hidden in the drawing. 
wire. At higher frequencies, some adjustments (linear scaling) of the dimensions of the wires are possible and at this stage we see no specific reason to use different polarizers. The dimensions of the polarizer must be selected to avoid interference between the signal and the WGP's frame. For example, in the case presented (Fig. 7), taking into account $20 \mathrm{~mm}$ diameter output channels and WGP positioned at $45^{\circ}$ towards the signal beam, a WGP with a diameter of at least $25 \mathrm{~mm}$ is needed.

\section{4. cSPr-output channels}

The bunch profile reconstruction depends on accurate measurements of the spectrum of the cSPr. It is only possible to measure the spectrum at a set of discrete frequencies with further interpolation and extrapolation generating a continuous spectrum from 0 to infinity $[9,32-$ 34]. Normally, the extrapolation to zero is done by using a Gaussian approximation and to infinity using polynomial functions $[9,34]$. There are a number of ways to select the sequence of the sampled frequencies and such a sequence depends on the specific design of the accelerator and expected bunch profile. If, for example, a singularity in the spectrum is expected, from the predictions during the accelerator design stage, one could consider more sampling points around the expected singularity using a sequence such as shown in (8) [33]:

$$
S_{m}=\sum_{m=0}^{m-1} a r^{m}=\frac{a\left(1-r^{m}\right)}{1-r}
$$

where $S_{m}$ is the frequency of the sample $m, a$ is the frequency position of the first detector and $r$ is the normalized parameter which controls the spacing between the samples. Alternatively, the set of the sampled frequencies can be equidistant in the frequency space, or angularly equidistant as in the current design [Fig. 4(b)]. The sequence of the sample frequencies will define the positions of the output channels along the arcs. In general, a larger number of the detectors (sampling frequencies) is beneficial for more accurate reconstruction of the profile [33]. The studies carried out in [33] assumed an initial Gaussian profile (with rms $\sigma_{0}$ ) and then deviations from this profile, both in length (from $0.1 \sigma_{0}$ to $10 \sigma_{0}$ ) and in shape. The changes in shape were simulated by differing values of $\sigma$ in the leading and trailing parts of the bunch. In each case, the accuracy of the reconstruction was tested as a function of the frequency sampling points and of the expected experimental uncertainty, or noise, in the measurements. The results indicate that in the "low noise" scenario, noise level of $\sim 1 \%$, five sampling points would give good reconstructions, with errors below $10 \%$. If the noise level is around 25\%, 35 sampling points would be required in order to achieve similar accuracy in the reconstruction.

\section{D. cSPr DoP and background signal subtraction}

In Fig. 7, the 3D drawing of one of the output channels of the monitor, capable of measuring both the signal intensity and the degree of polarization (DoP) at the sample frequency is shown. We define that the subchannel A will measure the intensity $S_{a}=I_{\|}$while the subchannel B will measure the intensity $S_{b}=I_{\perp} ; I_{\|, \perp}$ are the intensities of cSPr with electric fields parallel and perpendicular to the eigenvector of the corrugation of the grating. The degrees of polarization (DoP) from a specific grating $D_{\mathrm{sp}}$ or background $D_{N}$ are defined as

$$
D=\frac{I_{\|}-I_{\perp}}{I_{\|}+I_{\perp}}=\frac{I_{\|}-I_{\perp}}{I_{\text {tot }}},
$$

where $I_{\|, \perp}=k_{\|, \perp} I_{\text {tot }}$ and $I_{\text {tot }}=I_{\|}+I_{\perp}=\left(k_{\|}+k_{\perp}\right) I_{\text {tot }}$. The background radiation can be assumed to be unpolarized $[7,9,14,16,32-35]$ or alternatively its degree of polarization $D_{N}$ can be measured during the monitor installation. If the properties $D_{N, \text { sp }}$ of the background and coherent SP radiation are known and the signals $S_{a, b}$ are measured, the total intensity of cSPr can be calculated:

$$
I_{\mathrm{tot}}^{\mathrm{SP}}=\frac{S_{a}\left(1-D_{N}\right)-S_{b}\left(1+D_{N}\right)}{D_{\mathrm{sp}}-D_{N}}
$$

The simplest case is, if the background signals is unpolarized $D_{N}=0$ and $\mathrm{cSPr}$ is fully polarized $D_{\mathrm{sp}}=1$. In this case the cSPr total intensity can be evaluated from (10) as [7,9,14,16,32-35]

$$
I_{\mathrm{tot}}^{\mathrm{SP}}=S_{a}-S_{b} .
$$

The sources of the background and SP radiations are different and therefore the degree of polarization will be different at each sampling frequency allowing the separation of the cSPr from the background radiation. If at some frequencies the DoPs for the cSPr and background radiations are the same or due to noise cannot be distinguished, it would be impossible to distinguish the cSPr signal from the background radiation with this technique. In this case new sampling frequencies should be selected. As a result, understanding the properties of the cSPr and the background radiation is important, in particular when selecting an appropriate set of sampling frequencies. The studies of the cSPr degree of polarization are continuing and both 

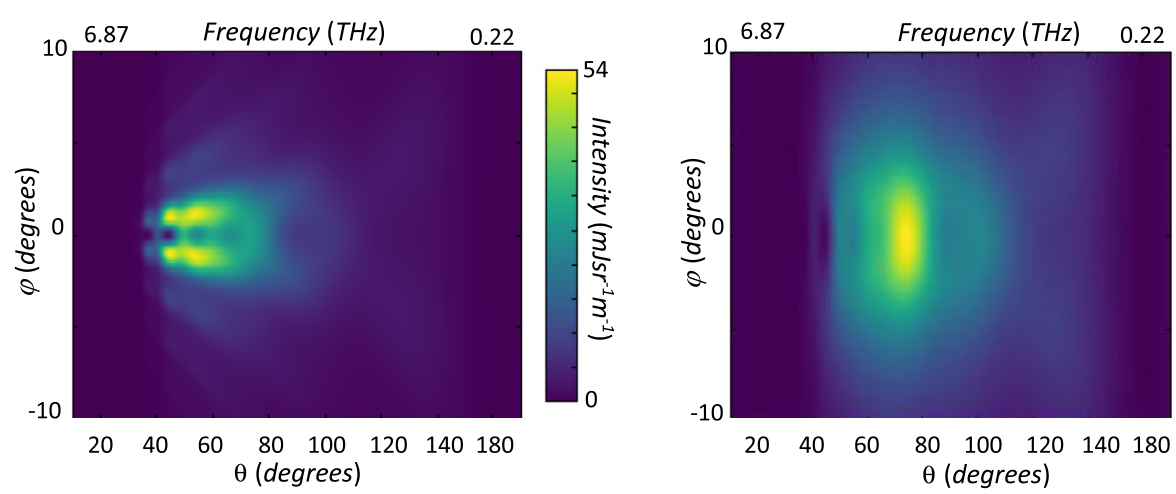

(a)
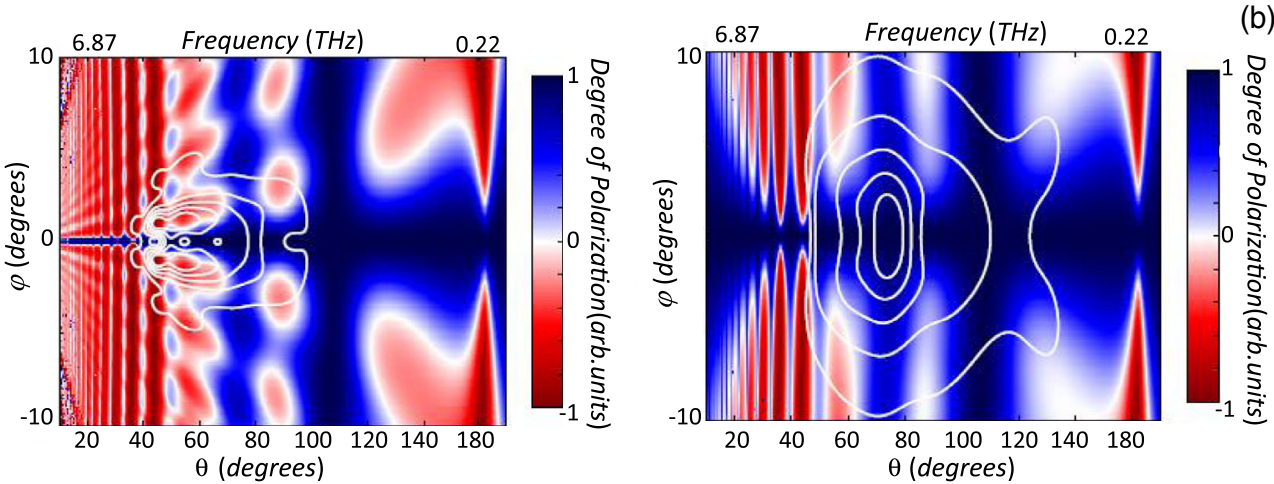

FIG. 8. The contour plots of (a) the intensities and (b) DoPs of cSPr signals over a range of observation angles $(\theta, \varphi)$ predicted from the $1 \mathrm{~mm}$ period grating if $1.2 \mathrm{nC}, 450 \mathrm{fs}$ electron bunch propagates above it. The contours in (b) indicate the equal amplitude radiation intensity distributions, showing the intensity levels, as seen in (a), from the lowest to highest value with $15 \%$ step increase for each new interval. The left and the right figures were derived for an electron energy of 20.35 and $10 \mathrm{GeV}$, respectively.

numerical and semianalytical models have been developed. The experimental studies of DoP [32-35] require a complex experimental setup and are still under development. These studies should be carried out for a single bunch and require measurements of the polarization of the EM radiation at $\mathrm{THz}$ frequencies at several sampling frequencies, using apparatus which does not interfere with the polarization of the signal. The theoretical studies show that in the case of finite dimensions of the output channel acceptance window the DoP of the cSPr depends on the detectors' coordinates, the channel aperture, bunch energy, the geometry of the grating, and the beam-grating separation distance. Figure 8 shows contour plots of the intensity $I_{\text {tot }}^{\mathrm{SP}}$ of the cSPr [Fig. 8(a)] and the degree of polarizations [Fig. 8(b)] $D_{\text {sp }}$ expected for $23 \mathrm{GeV}$ (left-hand-side figures) and $10 \mathrm{Gev}$ (right-hand-side figures), $1.2 \mathrm{nC}, 100 \mu \mathrm{m}$ Gaussian electron bunches propagating above a $1 \mathrm{~mm}$ period, $60 \mathrm{~mm}$ long grating. These plots were generated using semianalytical GFW code [7,9,33]. The figures show that the $D_{\text {sp }}$ is a function of the azimuthal $\phi$ and polar $\theta$ angles. It is also clear that both the cSPr directionality and the degree of polarization $\left(D_{\mathrm{sp}}\right)$ strongly depend on the bunch energy. The cSPr has constant $D_{\text {sp }}=$ 1 (1 parallel $I_{\|}$and -1 perpendicular $I_{\perp}$ polarizations) for all values of $\theta$ only strictly at the $\phi=0$. As the cSPr intensity outside $\phi=0$ (where $D_{\mathrm{sp}} \neq 1$ ) can be high, in experimental conditions, with a finite signal acceptance angle, the $D_{\text {sp }}$ varies significantly and it is expected to be below 1. On the other hand a large acceptance angle of the detection channels may be desirable to increase signal intensity at the detector. Figure 9 shows the dependence of the weighted averages (over a $12 \mathrm{~mm}$ diameter aperture) of $I_{\mathrm{tot}}^{\mathrm{SP}}$ and $D_{\mathrm{sp}}^{\mathrm{det}}$ of the cSPr signal for electron bunches ranging in energies from $10 \mathrm{MeV}$ to $5 \mathrm{GeV}$. Taking into account the acceptance aperture radius $\left(r_{\text {app }}\right)$ of the collecting optics, which is located at a distance $R_{\text {app }}$ from the grating, the weighted average value for $D_{\mathrm{sp}}$ is calculated as

$$
D_{\mathrm{sp}}^{\mathrm{det}}=\iint_{-A / 2}^{A / 2} D I d \phi d \theta / \iint_{-A / 2}^{A / 2} I d \phi d \theta
$$

where $A=\operatorname{artan}\left(\frac{r_{\text {app }}}{R_{\text {app }}}\right)$ is the angular aperture of the output window at a given position and the integration is taken over the $(\phi ; \theta)$. While the strong variation of the DoP on the position of the detectors has been also observed experimentally [9,33-35], it is worth noting that 


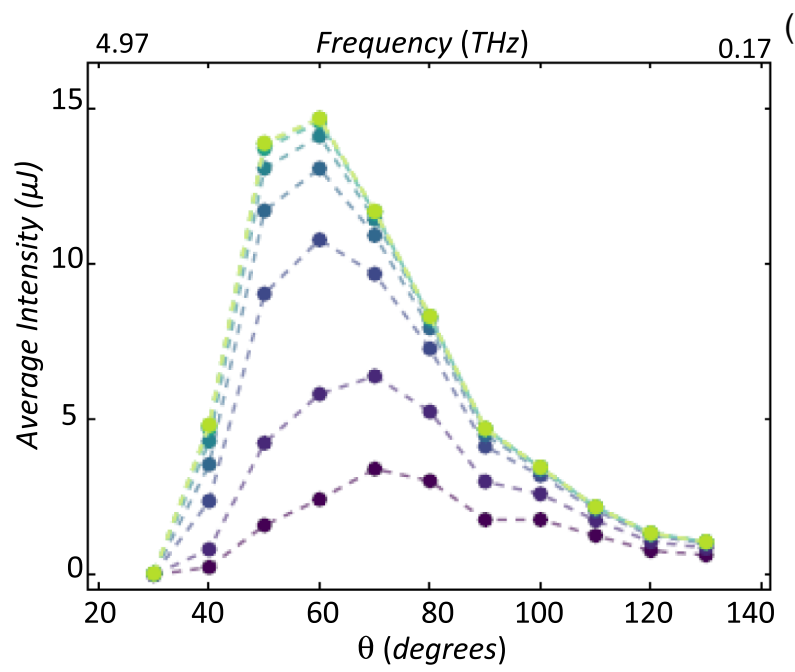

(a)

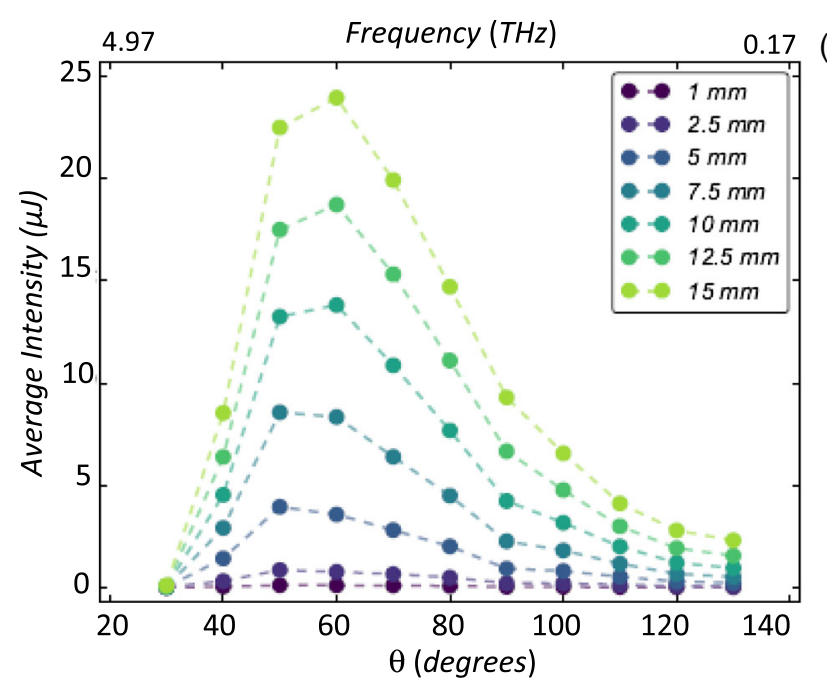

(a)
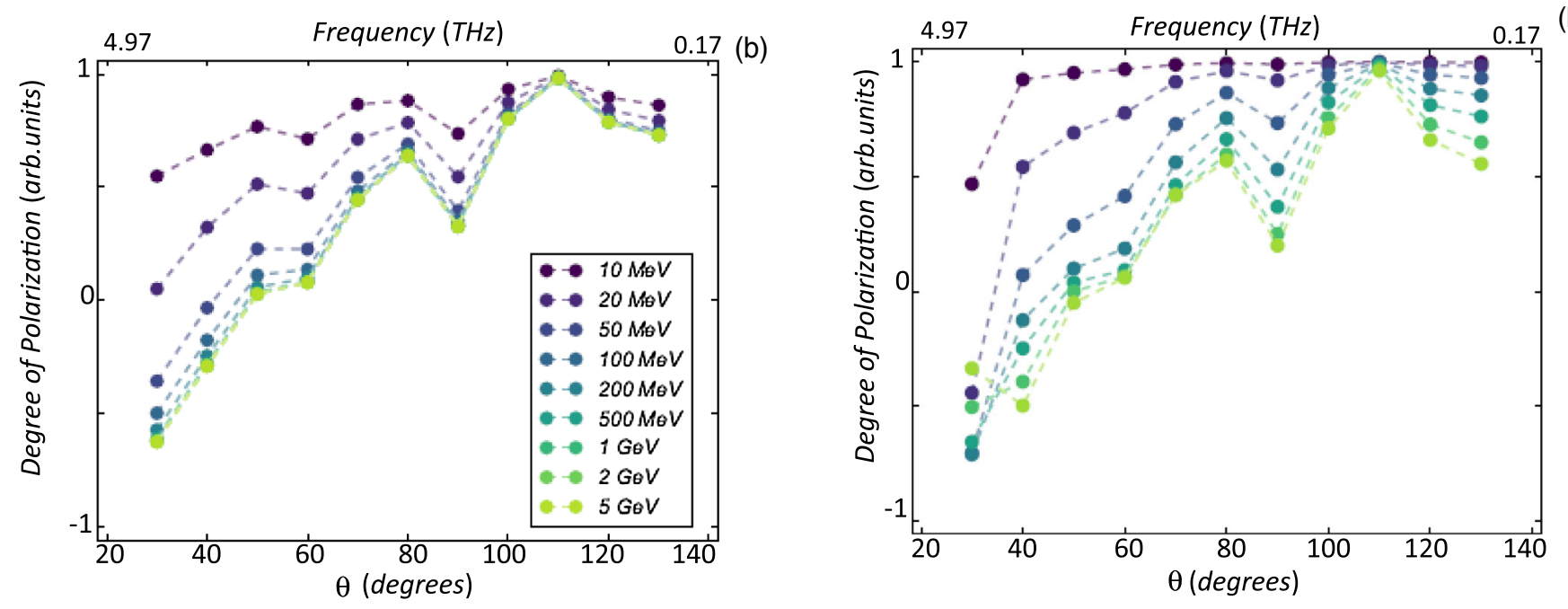

FIG. 9. Figures showing: (a) the calculated average intensity and (b) DoP of cSPr generated by electron bunches of varying energies, as shown in the inset of (b). The markers on each graph represent the location of the detectors along the $\theta$ coordinate (all detectors are located at $\varphi=0$ ). The values given for the average intensity have been integrated over the detector aperture.

conclusive and satisfactory experimental studies of the cSPr polarization are still to be carried out.

The oscillations of the DoP along the observation angles $\theta$ as seen in [9,33-35] can be avoided by the use of a specially designed antenna with a small aperture along $\phi$ with an estimated acceptance width below $5 \mathrm{~mm}$. Figure 10 shows in (a) the calculated average intensity and in (b) the DoP of cSPr calculated for varying detector acceptance apertures; it is clear that having small apertures may be beneficial. In this case it is noticeable that $D_{\text {sp }} \cong 1$ for all detectors for a broad range of beam energies and parameters. Another parameter which will affect the DoP is the grating profile. Carefully selecting the profile of the grating

will allow minimizing the DoP fluctuations, as shown in Fig. 11. The figures compare the DoP of the cSPr signals observed from sawtooth [Fig. 11(a)] and strip [Fig. 11(b)] gratings. In general the sawtooth grating has higher efficiency; however the DoP for the strip grating is flatter and higher. It has been established during the studies that the finite width of the grating and the finite width of the beam along $\phi$ will decrease the $D_{\text {sp }}$ below 1 . It has also been established that the beam charge does not directly affect the degree of the polarization. Knowing these properties of the cSPr polarization, as shown in Figs. 9-11, should allow one to design the gratings appropriately for a specific accelerator. 

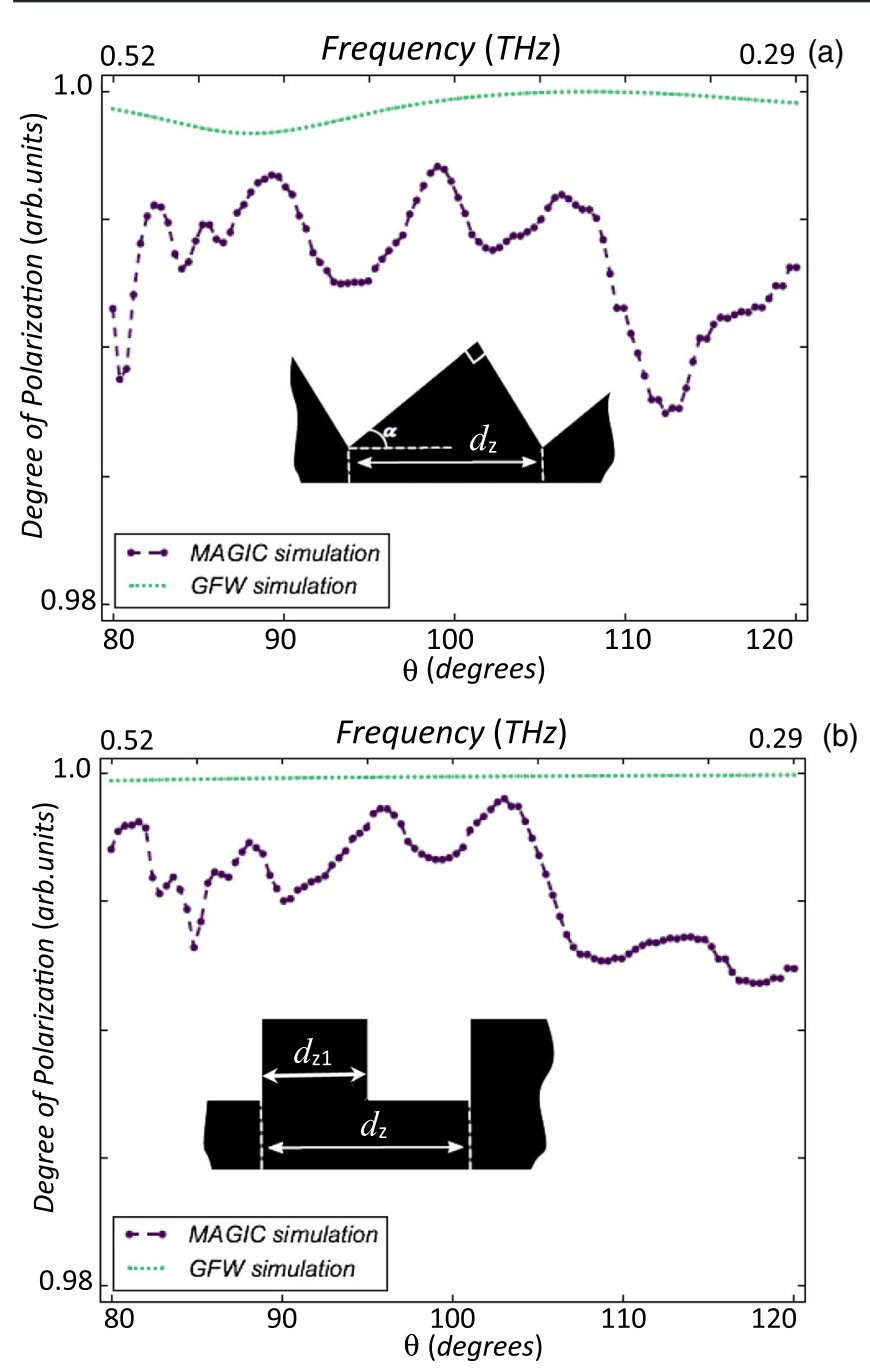

FIG. 11. The DoP of cSPr from the $10 \mathrm{~mm}$ long, $d_{z}=0.7 \mathrm{~mm}$ period grating obtained by using the semianalytical code GFW and the 3D particle-in-cell code MAGIC, for two different grating profiles: (a) sawtooth $\left(\alpha=30^{\circ}\right.$, height $0.25 \mathrm{~mm}$ see inset) and (b) strip (height $0.25 \mathrm{~mm}$ and $2 d_{z 1}=d_{z}$ see inset). The gratings were excited by $0.1 \mathrm{nC}, 8 \mathrm{MeV}, 300$ fs electron bunches. In both simulations it was assumed that the detectors were located at azimuthal angle $\varphi=0^{\circ}$ and the results were integrated over the detector aperture $(20 \mathrm{~mm}$ diameter).

\section{SUMMARY AND CONCLUSIONS}

The reconstruction of the temporal profile of a charged particle bunch is based on the analysis of the coherent radiation stimulated by the bunch. To achieve this, the radiation generated by the target has to be measured at a set of frequency sampling points and carefully separated from all background signals. After that, the full spectrum can be recovered by using extrapolation and interpolation techniques assuming that in the vicinity of the sampling points the spectrum is a smooth and well behaved function. The missing phase can be recovered and the bunch profile reconstructed either by using, for example,

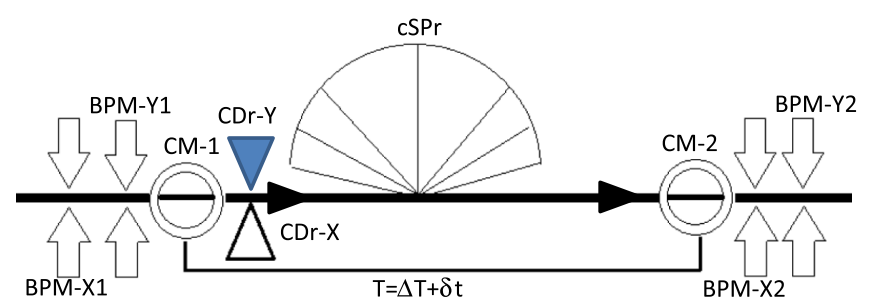

FIG. 12. A schematic of the conceptual design of the cSPr suite of the monitors which will enable 3D single shot, noninvasive, bunch image reconstruction. The suite includes: BPM- $(\mathrm{X}, \mathrm{Y})$ monitors, detuned by $\delta t$ bunch charge monitors $(\mathrm{CM}-1,2)$ and coherent diffraction radiation $(\mathrm{CDr}-\mathrm{X}, \mathrm{Y})$ monitors to measure the bunch transverse structure.

a phase-constrained iterative algorithm [34] or by the Kramers-Kronig method for the calculation of the minimal phase. In any case, it is essential to measure the spectrum accurately and over as broad a range as possible. Although in this paper we did not discuss the challenges associated with the phase reconstruction, a detailed discussion can be found in [34].

To operate the cSPr monitor and reconstruct the longitudinal profile of the bunch additional information such as the bunch charge, beam position (to determine beamgrating separation) and bunch transverse dimensions are required. This information is necessary for all techniques based on radiation spectrum analysis. Other information, for example the beam emittance, is also needed. However, the grating length is relatively small ( $40 \mathrm{~mm}$ in this case) and since we are primarily concerned with high energy beams $(\gamma \gg 1)$ the bunch width $\sigma_{x, y}$ will change slowly along the grating length $L_{z}$ even for beams of moderate emittance. Hence, it is beneficial to accompany the monitor with additional beam diagnostics, such as two BPMs up- and two BPMs downstream measuring $(x, y)$ at the beam entrance and the exit of the monitor and two coils monitoring the bunch charge up- and downstream. Having two charge monitors (CMs) as shown in Fig. 12 will also improve the bunch reconstruction technique if one of the monitors is slightly delayed and measures only part of the bunch charge. This can be achieved by delaying the triggering of the second monitor by $\delta t$ where $\delta t<\tau+\Delta T / 2, \tau$ is the transit time of the bunch between the two monitors and $\Delta T$ is the expected bunch length. The value of the first monitor will then provide the total charge of the bunch while the second monitor will provide the partial value of the bunch charge in the tail, helping for example to resolve the tail-head uncertainty and improve further the Kramers-Kronig phase limiting iterative reconstruction techniques [34]. Figure 12 shows the schematic of such an arrangement, with all the facilitating components which also includes additional optical diffraction radiation (ODR) screens. These screens can provide information about the transverse dimensions and transverse structure of the beam. As discussed above, such 
information is important for the determination of the DoP of the coherent SP radiation. The ODR monitors, in combination with cSPr longitudinal profile monitors, can be used to recover nondestructively in a single shot a full 3D image of the bunch [36].

In summary, this paper has described the design of a single shot bunch profile monitor based on coherent SmithPurcell radiation and capable of determining in a nondestructive way the longitudinal profile of a single bunch. In addition, it can monitor the longitudinal microbunching (spacing and degree of modulation) of a charged particle beam and study the properties of coherent Smith-Purcell radiation. The design is based on the results of comprehensive theoretical and experimental studies of the multishot bunch profile monitor and the properties of cSPr carried out at FACET (SLAC, USA), LUCX (KEK, Japan) and Tsinghua University (China). The operational principles of the monitor were discussed and its essential components were presented. Design challenges associated with each component such as vacuum chamber, number of output channels and detectors and their positioning, were also discussed. It was shown that the degree of experimental uncertainty in the measurements affects the number of frequency sampling points required. The number of the detection channels in the present design (33) should provide good reconstruction even when the uncertainty in the data is of the order of $25 \%$. Accurate knowledge of the degree of polarization of the cSPr is essential for the separation of this signal from the background radiation. This monitor should be seen as part of a suite of diagnostic devices that will determine other parameters of the bunch, such as charge, transverse size etc. We have indicated possible future developments and the most interesting steps to improve the monitor performance. The studies show that the present design can be adapted to a broad range of accelerators operating within the energy range from $8 \mathrm{MeV}$ to $23 \mathrm{GeV}$ and capable of generating fs-long bunches. The next steps of the monitor development will be construction and its installation on an accelerator beam line, followed by rigorous studies of the cSPr polarization properties and demonstration of the single shot capabilities, including the capability to monitor the beam microbunching and bunchbunch separation. The construction and test of the monitor will lead to a better understanding of beam dynamics as well as improve the understanding of the operation of the next generation of light sources.

\section{ACKNOWLEDGMENTS}

The authors would like to thank STFC UKRI for support of these studies via JAI Grants No. ST/P002048/1 and No. ST/P000967/1, STFC PRD Grant No. ST/M003590/1 and STFC Grant No. ST/S002227/1. Professor I. Konoplev would like to thank Leverhulme Trust for supporting the research network via Grant No. IN-2015-012 and Royal Society of London (U.K.) for supporting travel via U.K.-
Japan exchange Grant No. IEC\R3\170010. Dr. Hannah Harrison would like to thank STFC U.K. and JAI for supporting her D.Phil. project at the Department of Physics, University of Oxford. Dr. Huibo Zhang would like to thank JAI and the Chinese Government for supporting his D.Phil. project at the Department of Physics, University of Oxford.

\section{APPENDIX A: DESIGN OF THE 2D GRATINGS}

One way to improve the measurements of the spectrum is to design the grating (1) carefully. The grating design can also allow the simplification of the monitor structure and resolve issues with signal diffraction due to the finite width of the grating.

One of the grating parameters is the amplitude of the corrugation. It is always assumed to be small, meaning that the periodic patterns can be Fourier decomposed and presented as superposition of elementary gratings $[18,19,22,23]$ (linearity of the gratings). This also means that the surface currents induced on one of the facets of the grating are not interacting with the surface currents induced on the other facet via self-fields (noncommunicative model) and the total signal radiated is a superposition of the signals from each elementary facet/grating (linearity of the radiated signals). This opens up the possibility to consider a complex 2D patterns (1) i.e., superposition of several (let us say M) gratings of different periodicities $\bar{k}_{z, i}, \bar{k}_{y, j}[18,19,23]$, for a single grating $i=j$. The expression (1) is the Fourier decomposition of any complex 2D patterns into "elementary" gratings and the linearity allows one to consider each grating separately while the total response, in the far field zone, will be a superposition of the signals' complex amplitudes generated by each individual elementary grating. The design of such complex patterns is an interesting and nontrivial challenge and the representation of such complex patterns through a set of elementary gratings significantly simplifies the task. The use of the complex patterns (2D patterns) will allow an increase in the number of sampling frequencies as well as in the frequency range covered by a single target. In this case, the separation of the cSPr signals observed from each elementary grating (tuned to cover a specific frequency region) can be achieved via slight tilting of each elementary grating by angle $\phi_{i}$ where $i$ indicates the number of the grating. Let us consider as a simple example a grating which has $\bar{k}_{y, i} \neq 0, \bar{k}_{z, i} \neq 0$ i.e., it is "tilted" by an angle $\tilde{\phi}\left(\operatorname{tg}(\tilde{\phi})=\frac{\bar{k}_{y}}{k_{z}}\right)$ with respect to the direction of the beam propagation. The tilt effectively changes the grating period along $z$ (as compared with grating of the same period and $\bar{k}_{y, i}=0$ ) resulting in the change of the operating frequency range and a change in the directionality of the radiation as $\bar{k}_{y, i} \neq 0$ (no "crosstalking"). The distribution of the maxima of the intensities of such a tilted grating can be evaluated from consideration of the grating as a superposition of two elementary 
diffraction gratings [23], the first with $\bar{k}_{y}=0, \bar{k}_{z} \neq 0$ and the second with $\bar{k}_{y} \neq 0, \bar{k}_{z}=0$. For the first grating the term $\cos \tilde{\phi}$ should be added to the dispersion relation (1) to account for the grating's period change $\left(d=d_{z} / \cos \tilde{\phi}\right)$ :

$$
\lambda=\frac{d_{z}}{n \cos \tilde{\phi}}\left(\frac{1}{\beta_{z}}-\cos \theta\right)
$$

while the second grating is a normal diffraction grating for which the conventional relation is satisfied:

$$
\lambda=\frac{d_{z} \sin \theta \sin \phi}{m \sin \tilde{\phi}},
$$

where $m$ is also harmonic number and $m \neq n$. Equating expressions (A1) and (A2) will give us the relation which links the location of the intensity maximum in $\phi$ with the grating wave numbers $\bar{k}_{y}, \bar{k}_{z}$ :

$$
\sin \phi=\frac{m \bar{k}_{y}}{n \bar{k}_{z} \sin \theta(\lambda)}\left(\frac{1}{\beta_{z}}-\cos \theta(\lambda)\right) .
$$

We note that (A3) depends on the radiated wavelength as $\theta(\lambda)$ which is defined by (A1). As a result the linearity of the shallow gratings allows location of several corrugations [23] on one target, broadening the operating frequency range and the possible number of a sampling frequencies. It should be remembered that the gratings should be of similar period to ensure that the beam grating separation is appropriate for all of them. There are a number of other issues which may also create some challenges in the implementation of such a target, including directionality of the secondary lobs of the radiation and complexity of the patterns. As a result, the detailed design of the target with the complex 2D patterns for cSPr monitor requires careful consideration in the future and is outside the scope of this paper.

As mentioned in Sec. III A, the use of wide gratings is advisable in order to minimize the interference from the grating boundaries [Fig. 2(b)]. The finite dimensions of the grating result in loss of efficiency, the appearance of high order transverse modes [Fig. 2(b)] and signal angular dispersion which can be estimated as $\delta \hat{\varphi} \sim \lambda / 2 L_{w}$. This angular dispersion (due to finite grating width) can be overcome by using curved gratings with the curvature radius in the region $2 L_{g r}^{2} / \lambda>R \geq 2 L_{g r} L_{w} / \lambda$, where $L_{g r}$ is the length of the grating. In this case the focal point will be in front of the detectors (avoiding overfocusing) whose positions are defined by the far field zone condition $d>\frac{2 L_{g r}^{2}}{\lambda}$. Considering the development of new manufacturing technologies [18] the construction of such complex gratings should not be a challenge, especially in the $\mathrm{THz}$ frequency range.
[1] R. Farias, S. Rimjaem, C. Settakorn, T. Vilaithong, and H. Wiedemann, Production and use of femtosecond electron bunches, edited by $\mathrm{H}$. Wiedemann, Electron-Photon Interaction in Dense Media. NATO Science Series (Series II: Mathematics, Physics and Chemistry) (Springer, Dordrecht, 2002), Vol. 49; K. Nakajima, Laser-driven electron beam and radiation sources for basic, medical and industrial sciences, Proc. Jpn. Acad. Ser. B 91, 223 (2015); R. Bingham, Basic concepts in plasma accelerators, Phil. Trans. R. Soc. A 364, 559 (2006).

[2] M. Boscolo, M. Ferrario, I. Boscolo, F. Castelli, and S. Cialdi, Generation of short $\mathrm{THz}$ bunch trains in a $\mathrm{rf}$ photoinjector, Nucl. Instrum. Methods Phys. Res., Sect. A 577, 409 (2007); E. A. Nanni, W. R. Huang, K. H. Hong, K. Ravi, A. Fallahi, G. Moriena, R. J. Miller, and F. X. Kärtner, Terahertz-driven linear electron acceleration, Nat. Commun. 6, 8486 (2015).

[3] T. Plath, C. Lechner, V. Miltchev, P. Amstutz, N. Ekanayake, L. L. Lazzarino, T. Maltezopoulos, J. Bödewadt, T. Laarmann, and J. Roßbach, Mapping few-femtosecond slices of ultrarelativistic electron bunches, Sci. Rep. 7, 2431 (2017).

[4] E. Prat, P. Dijkstal, M. Aiba, S. Bettoni, P. Craievich, E. Ferrari, R. Ischebeck, F. Löhl, A. Malyzhenkov, G. L. Orlandi, S. Reiche, and T. Schietinger, Generation and Characterization of Intense Ultralow-Emittance Electron Beams for Compact X-Ray Free-Electron Lasers, Phys. Rev. Lett. 123, 234801 (2019).

[5] E. Brunetti, R. P. Shanks, G. G. Manahan, M. R. Islam, B. Ersfeld, M. P. Anania, S. Cipiccia, R. C. Issac, G. Raj, G. Vieux, and G. H. Welsh, S.M. Wiggins, and D. A. Jaroszynski, Low Emittance, High Brilliance Relativistic Electron Beams from a Laser-Plasma Accelerator, Phys. Rev. Lett. 105, 215007 (2010); B. Hermann, S. Bettoni, T. Egenolf, U. Niedermayer, E. Prat, and R. Ischebeck, Laserdriven modulation of electron beams in a dielectric microstructure for x-ray free-electron lasers, Sci. Rep. 9, 19773 (2019).

[6] K. K. Kainz, K. R. Hogstrom, J. A. Antolak, P. R. Almond, C. D. Bloch, C. Chiu, M. Fomytskyi, F. Raischel, M. Downer, and T. Tajima, Dose properties of a laser accelerated electron beam and prospects for clinical application, Med. Phys. 31, 2053 (2004); M. Oppelt, M. Baumann, R. Bergmann, E. Beyreuther, K. Brüchner, J. Hartmann, L. Karsch, M. Krause, L. Laschinsky, E. Leßmann, M. Nicolai, M. Reuter, C. Richter, A. Sävert, M. Schnell, M. Schürer, J. Woithe, M. Kaluza, and J. Pawelke, Comparison study of in vivo dose response to laser-driven versus conventional electron beam, Radiat. Environ. Biophys. 54, 155 (2015).

[7] G. Doucas, M. F. Kimmitt, A. Doria, G. P. Gallerano, E. Giovenale, G. Messina, H. L. Andrews, and J. H. Brownell, Determination of longitudinal bunch shape by means of coherent Smith-Purcell radiation, Phys. Rev. ST Accel. Beams 5, 072802 (2002); G. Naumenko, B. Kalinin, G. Saruev, D. Karlovets, Yu. Popov, A. Potylitsyn, L. Sukhikh, and V. Cha, Characteristics of Smith-Purcell radiation from different profile gratings, in Proceedings of the 29th Free Electron Laser Conference, Novosibirsk, Russia (BINP, Novosibirsk, 2007), WEPPH055, 
pp. 480-483; V. Blackmore, G. Doucas, C. Perry, B. Ottewell, M. F. Kimmitt, M. Woods, S. Molloy, and R. Arnold, First measurements of the longitudinal bunch profile of a $28.5 \mathrm{GeV}$ beam using coherent Smith-Purcell radiation, Phys. Rev. ST Accel. Beams 12, 032803 (2009).

[8] W. Barry, Measurement of subpicosecond bunch profiles using coherent transition radiation, AIP Conf. Proc. 390, 173 (1997); M. Heigoldt, A. Popp, K. Khrennikov, J. Wenz, S. W. Chou, S. Karsch, S. I. Bajlekov, S. M. Hooker, and B. Schmidt, Temporal evolution of longitudinal bunch profile in a laser wakefield accelerator, Phys. Rev. ST Accel. Beams 18, 121302 (2015); D. Mihalcea, C. L. Bohn, U. Happek, and P. Piot, Longitudinal electron bunch diagnostics using coherent transition radiation, Phys. Rev. ST Accel. Beams 9, 082801 (2006).

[9] H. L. Andrews, F. B. Taheri, J. Barros et al., Reconstruction of the time profile of $20.35 \mathrm{GeV}$, subpicosecond long electron bunches by means of coherent SmithPurcell radiation, Phys. Rev. ST Accel. Beams 17, 052802 (2014); H. L. Andrews, F. B. Taheri, J. Barros et al., Longitudinal profile monitors using Coherent SmithPurcell radiation, Nucl. Instrum. Methods Phys. Res., Sect. A 740, 212 (2014).

[10] C. P. Welsch, H. H. Braun, E. Bravin, R. Corsini, S. Döbert, T. Lefèvre, F. Tecker, P. Urschütz, B. Buonomo, O. Coiro, A. Ghigo, and B. Preger, Longitudinal beam profile measurements at CTF3 using a streak camera, J. Instrum. 1, P09002 (2006); W. Cheng, B. Bacha, A. Blednykh, Y. $\mathrm{Li}$, and O. Singh, Longitudinal bunch profile measurement at NSLS2 storage ring, in Proceedings of IBIC2015, Melbourne, Australia, 2015, MOPB083 (2015), pp. 251-255; A. E. Dabrowski, E. Adli, S. Bettoni et al., Measuring the longitudinal bunch profile at CTF3, in Proceedings of the 25th International Linear Accelerator Conference, LINAC-2010, Tsukuba, Japan (KEK, Tsukuba, Japan, 2010), TUP100, pp. 647-649.

[11] G. Berden, A. F. G. van der Meer, S. P. Jamison, B. Steffen, E.-A. Knabbe, B. Schmidt, P. Schmuser, A. M. MacLeod, P. J. Phillips, and W. A. Gillespie, Single shot longitudinal bunch profile measurements at FLASH using electrooptic techniques, in Proceedings of the 10th European Particle Accelerator Conference, Edinburgh, Scotland, 2006 (EPS-AG, Edinburgh, Scotland, 2006), TUPCH026, pp. 1055-1057; R. Pompili, A. Cianchi, D. Alesini et al., First single shot and nonintercepting longitudinal bunch diagnostics for comb-like beam by means of electro-optic sampling, Nucl. Instrum. Methods Phys. Res., Sect. A 740, 216 (2014); B. Steffen, M. K. Czwalinna, Ch. Gerth, and P. Peier, First electro-optical bunch length measurements at the European XFEL, in the 7th International Beam Instrumentation Conference, IBIC 2018 (JACoW Publishing, Shanghai, China, 2018), pp. 338-341; S. Funkner, E. Blomley, E. Bründermann et al., High throughput data streaming of individual longitudinal electron bunch profiles, Phys. Rev. Accel. Beams 22, 022801 (2019).

[12] O. Lundh, C. Rechatin, J. Lim, V. Malka, and J. Faure, Experimental Measurements of Electron-Bunch Trains in a Laser-Plasma Accelerator, Phys. Rev. Lett. 110, 065005 (2013); A. Marinelli, M. Dunning, S. Weathersby, E. Hemsing, D. Xiang, G. Andonian, F. O’Shea, J. Miao,
C. Hast, and J. B. Rosenzweig, Single-Shot Coherent Diffraction Imaging of Microbunched Relativistic Electron Beams for Free-Electron Laser Applications, Phys. Rev. Lett. 110, 094802 (2013); R. B. Fiorito, C. P. Welsch, A. G. Shkvarunets, C. Clarke, and A. Fisher, Coherent diffraction radiation imaging methods to measure rms bunch, in Proceedings of IBIC2016 (JACow Publishing, Barcelona, Spain, 2016), MOPG58, pp. 198-200.

[13] A. Curcio, M. Bergamaschi, R. Corsini et al., Noninvasive bunch length measurements exploiting Cherenkov diffraction radiation, Phys. Rev. Accel. Beams 23, 022802 (2020).

[14] J. H. Brownell, J. Walsh, and G. Doucas, Spontaneous Smith-Purcell radiation described through induced surface currents, Phys. Rev. E 57, 1075 (1998); E. B. Schmidt, S. Wesch, T. Kovener, C. Behrens, E. Hass, S. Casalbuoni, and P. Schmuser, Longitudinal bunch diagnostics using coherent transition radiation spectroscopy physical principles, multichannel spectrometer, experimental results, mathematical methods, arXiv:1803.00608v1; J. Thangaraj, G. Andonian, R. Thurman-Keup, J. Ruan, A. S. Johnson, A. Lumpkin, J. Santucci, T. Maxwell, A. Murokh, M. Ruelas, and A. Ovodenko, Demonstration of a real-time interferometer as a bunch-length monitor in a high-current electron beam accelerator, Rev. Sci. Instrum. 83, 043302 (2012).

[15] B. Kehrer, E. Blomley, M. Brosi et al., Simultaneous detection of longitudinal and transverse bunch signals at ANKA, in Proceedings of IPAC2016, Busan, Korea, 2016, http://accelconf.web.cern.ch/ipac2016/papers/mopmb014 .pdf, MOPMB014, pp. 109-111.

[16] G. Doucas, V. Blackmore, B. Ottewell, C. Perry, P. G. Huggard, E. Castro-Camus, M. B. Johnston, J. Lloyd Hughes, M. F. Kimmitt, B. Redlich, and A. van der Meer, Longitudinal electron bunch profile diagnostics at $45 \mathrm{MeV}$ using coherent Smith-Purcell radiation, Phys. Rev. ST Accel. Beams 9, 092801 (2006); V. Khodnevych, N. Delerue, S. Jenzer, and H. Roesch, Study of a SmithPurcell radiation-based longitudinal profile monitor at the CLIO free electron laser, in Proceedings of IPAC2017, Copenhagen, Denmark, 2017, MOPAB026, pp. 132-134, https://doi.org/10.18429/JACoW-IPAC2017-MOPAB026; N. Deleruey, S. Jenzer, V. Khodnevych, A. Migayron, J.-P. Berthet, N. Jestin, J.-M. Ortega, and R. Prazeres, Bunch length measurements using coherent Smith-Purcell radiation with several gratings at CLIO, in the 9th International Particle Accelerator Conference, IPAC2018 (JACoW Publishing, Vancouver, BC, Canada, 2018), https:// doi.org/10.18429/JACoW-IPAC2018-WEPAL034.

[17] C. M. Scoby, R. K. Li, E. Threlkeld, H. To, and P. Musumeci, Single-shot 35 fs temporal resolution electron shadowgraphy, Appl. Phys. Lett. 102, 023506 (2013); A. Sävert, S. P. D. Mangles, M. Schnell et al., Direct Observation of the Injection Dynamics of a Laser Wakefield Accelerator Using Few-Femtosecond Shadowgraphy, Phys. Rev. Lett. 115, 055002 (2015); M. C. Downer, R. Zgadzaj, A. Debus, U. Schramm, and M. C. Kaluza, Diagnostics for plasma-based electron accelerators, Rev. Mod. Phys. 90, 035002 (2018); R. Tarkeshian, J. L. Vay, R. Lehe, C. B. Schroeder, E. H. Esarey, T. Feurer, and W. P. Leemans, Transverse 
Space-Charge Field-Induced Plasma Dynamics for Ultraintense Electron-Beam Characterization, Phys. Rev. X 8, 021039 (2018).

[18] I. V. Konoplev, L. Fisher, K. Ronald, A. W. Cross, A. D. R. Phelps, C. W. Robertson, and M. Thumm, Surface-field cavity based on a two-dimensional cylindrical lattice, Appl. Phys. Lett. 96, 231111 (2010); A. R. Phipps, A. J. MacLachlan, C. W. Robertson, L. Zhang, I. V. Konoplev, A. W. Cross, and A. D. R. Phelps, Electron beam excitation of coherent sub-terahertz radiation in periodic structures manufactured by 3D printing, Nucl. Instrum. Methods Phys. Res., Sect. B 402, 202 (2017).

[19] I. V. Konoplev, A. W. Cross, and A. D. R. Phelps, Relativistic electron beam excitation of surface fields in artificial materials based on one- and two-dimensional periodic structures, IEEE Trans. Plasma Sci. 39, 2610 (2011).

[20] P. Schoessow, M. E. Conde, W. Gai, R. Konecny, J. Power, and J. Simpson, High power radio frequency generation by relativistic beams in dielectric structures, J. Appl. Phys. 84, 663 (1998); J. A. Felix, J. R. Schwank, D. M. Fleetwood, M. R. Shaneyfelt, and E. P. Gusev, Effects of radiation and charge trapping on the reliability of high-jgate dielectrics, Microelectron. Reliab. 44, 563 (2004).

[21] Y. Liang, Y. Du, D. Wang et al., Selective excitation and control of coherent terahertz Smith-Purcell radiation by high-intensity period-tunable train of electron microbunches, Appl. Phys. Lett. 113, 171104 (2018).

[22] I. V. Konoplev, P. MacInnes, A. W. Cross, A. D. R. Phelps, and K. Ronald, Study of one-dimensional Bragg structures with localized defect, Appl. Phys. Lett. 91, 171107 (2007); I. V. Konoplev, G. Doherty, A. W. Cross, A. D. R. Phelps, and K. Ronald, Photonic band gap control in one-dimensional dielectric Bragg structures, Appl. Phys. Lett. 88, 111108 (2006).

[23] A. W. Cross, I. V. Konoplev, A. D. R. Phelps, and K. Ronald, Studies of surface two-dimensional photonic band-gap structures, J. Appl. Phys. 93, 2208 (2003); A. J. Lancaster, G. Doucas, H. Harrison, and I. V. Konoplev, Novel grating designs for a single shot Smith-Purcell bunch profile monitor, in Proceedings of IBIC2016, International Beam Instrumentation Conference (JACoW Publishing, Barcelona, Spain, 2016), MOPG62 A. J. MacLachlan, C. W. Robertson, I. V. Konoplev, A. W. Cross, A. D. R. Phelps, and K. Ronald, Resonant excitation of volume and surface fields on complex electrodynamic surfaces, Phys. Rev. Applied 11, 034034 (2019).

[24] B. Goplen, L. Ludeking, D. Smithe, and G. Warren, Userconfigurable MAGIC code for electromagnetic PIC calculations, Comput. Phys. Commun. 87, 54 (1995).

[25] Z. Shi, Z. Yang, Z. Liang, D. Li, and K. Imasaki, Superradiant smith-purcell radiation in the terahertz-wave region from bunched electron beams, Proceedings of FEL 2006, BESSY, Berlin, Germany (2006), TUPPH048, pp. 435438, https://accelconf.web.cern.ch/f06/PAPERS/ TUPPH048.PDF; J. T. Donohue and J. Gardelle, Simulation of a Smith-Purcell free-electron laser with sidewalls: Copious emission at the fundamental frequency, Appl. Phys. Lett. 99, 161112 (2011).

[26] H. Zhang, I. V. Konoplev, A. J. Lancaster, H. Harrison, G. Doucas, A. Aryshev, M. Shevelev, N. Terunuma, and
J. Urakawa, Nondestructive measurement and monitoring of separation of charged particle microbunches, Appl. Phys. Lett. 111, 043505 (2017); Y. Liang, Y. Du, X. Su et al., Observation of coherent Smith-Purcell and transition radiation driven by single bunch and microbunched electron beams, Appl. Phys. Lett. 112, 053501 (2018); G. Liao, Y. Li, H. Liu et al., Multimillijoule coherent terahertz bursts from picosecond laser-irradiated metal foils, Proc. Natl. Acad. Sci. U.S.A. 116, 3994 (2019).

[27] I. V. Konoplev, D. W. P. De Boer, C. Warsop, and M. John, Beam impedance minimization for accelerator beam line insertion devices, Rev. Sci. Instrum. 91, 074711 (2020).

[28] C. Winnewisser, F. Lewen, and H. Helm, Transmission characteristics of dichroic filters measured by $\mathrm{THz}$ timedomain spectroscopy, Appl. Phys. A 66, 593 (1998); E. Yablonovitch, T. J. Gmitter, R. D. Meade, A. M. Rappe, K. D. Brommer, and J.D. Joannopoulos, Donor and Acceptor Modes in Photonic Band Structure, Phys. Rev. Lett. 67, 3380 (1991); D. W. Porterfield, J. L. Hesler, R. Densing, E. R. Mueller, T. W. Crowe, and R. M. Weikle, Resonant metal-mesh bandpass filters for the far infrared, Appl. Opt. 33, 6046 (1994).

[29] R. Winston and J. M. Enoch, Retinal cone receptor as an ideal light collector, J. Opt. Soc. Am. 61, 1120 (1971); M. Aoki, S. R. Tripathi, M. Takeda, and N. Hiromoto, Angle dependent condensing efficiency of Winston cone in terahertz region, Infrared Phys. Technol. 56, 8 (2013); E. N. Grossman, O. D. Friedman, and A. O. Nelson, Nonimaging Winston cone concentrators for submillimeterwave, overmoded waveguide, IEEE Trans. Terahertz Sci. Technol. 4, 65 (2014).

[30] N. Daghestani, K. Parow-Souchon, D. Pardo, H. Liu, N. Brewster, M. Frogley, G. Cinque, B. Alderman, and P. G. Huggard, Room temperature ultrafast InGaAs Schottky diode based detectors for terahertz spectroscopy, Infrared Phys. Technol. 99, 240 (2019); S. Preu, M. Mittendorff, S. Winnerl, O. Cojocari, and A. Penirschke, $\mathrm{THz}$ autocorrelators for ps pulse characterization based on Schottky diodes and rectifying field-effect transistors, IEEE Trans. Terahertz Sci. Technol. 5, 922 (2015); N. S. Daghestani, K. Parow-Souchon, D. Pardo, F. Cahill, M. D. Frogley, J. Langston, B. E. J. Alderman, G. Cinque, and P. G. Huggard, Linear detection of coherent synchrotron radiation emitted by single electron bunches using zero-biased InGaAs Schottky diode detectors, in the 43rd International Conference on Infrared, Millimeter, and Terahertz. Waves (IRMMW-THz), Nagoya, Japan, 2018, https://doi.org/10.1109/IRMMWTHz.2018.8510427.

[31] G. R. Bird and M. Parrish, The wire grid as a near-infrared polarizer, J. Opt. Soc. Am. 50, 886 (1960); P. Yeh, A new optical model for wire grid polarizers, Opt. Commun. 26, 289 (1978).

[32] H. Harrison, A. J. Lancaster, I. V. Konoplev, G. Doucas, A. Aryshev, M. Shevelev, N. Terunuma, J. Urakawa, and P. G. Huggard, A Fabry-Pérot interferometer with wiregrid polarizers as beamsplitters at terahertz frequencies, Rev. Sci. Instrum. 89, 035116 (2018). 
[33] H. Harrison, Design of single shot longitudinal bunch profile monitor based on analysis of coherent Smith-Purcell radiation, D.Phil. thesis, University of Oxford, 2018.

[34] F. B. Taheri, I. V. Konoplev, G. Doucas, P. Baddoo, R. Bartolini, J. Cowley, and S. M. Hooker, Electron bunch profile reconstruction based on phase-constrained iterative algorithm, Phys. Rev. Accel. Beams 19, 032801 (2016); B. Schmidt, N. M. Lockmann, P. Schmüser, and S. Wesch, Benchmarking coherent radiation spectroscopy as a tool for high-resolution bunch shape reconstruction at freeelectron lasers, Phys. Rev. Accel. Beams 23, 062801 (2020).

[35] F. B. Taheri, I. V. Konoplev, G. Doucas, A. Reichold, R. Bartolini, N. Delerue, J. Barros, and C. Clarke, Experimental and theoretical studies of the properties of coherent Smith-Purcell radiation, in Proceedings of the 7 th International Particle Accelerator Conference (IPAC
2016, Busan, Korea, 2016, MOPMR041, pp. 344-346, https://doi.org/10.18429/JACoW-IPAC2016-MOPMR041; F. B. Taheri, Numerical and experimental studies of coherent Smith-Purcell radiation, D.Phil. thesis, University of Oxford, 2016.

[36] Y. Okayasu, H. Tomizawa, S. Matsubara, N. Kumagai, A. Maekawa, M. Uesaka, and T. Ishikawa, Feasibility study of a single shot 3D electron bunch shape monitor with an electro-optic sampling technique, Phys. Rev. ST Accel. Beams 16, 052801 (2013); D. Marx, R. Assmann, P. Craievich, U. Dorda, A. Grudiev, and B. Mandarchetti, Reconstruction of the 3D charge distribution of an electron bunch using a novel variable-polarization transverse deflecting structure (TDS), in the 8th International Particle Accelerator Conference, Copenhagen, Denmark, 2017 [J. Phys. Conf. Ser. 874, 012077 (2017)]. 